\title{
An Easy Approach to Optically Active $\alpha$-Amino Phosphonic Acid Derivatives by Chiral Zn(II) Catalyzed Enantioselective Amination of Phosphonates
}

\author{
Luca Bernardi, Wei Zhuang, Karl Anker Jørgensen* \\ The Danish National Research Foundation: Center for Catalysis, Department of \\ Chemistry, Aarhus University, DK-8000 Aarhus C, Denmark, Fax (45) 89196199 \\ e-mail:kaj@.chem.au.dk
}

\section{Supporting Information}

\section{Contents:}

- Experimental Section

- Representative Results from the Screening of Different Catalysts, Solvents and Additives for the Amination Reaction

- NMR Spectra and HPLC Chromatograms for the Products

- Absolute Configuration and Proposed Intermediate 


\section{Experimental Section.}

General Methods. ${ }^{1} \mathrm{H}$ and ${ }^{13} \mathrm{C}$ NMR spectra were recorded on a Varian AS 400 spectrometer, running at $400 \mathrm{MHz}$ and $100 \mathrm{MHz}$, respectively, in $\mathrm{CDCl}_{3}$ as the solvent Chemical shifts were reported in the $\delta$ scale relative to residual $\mathrm{CHCl}_{3}(7.26 \mathrm{ppm})$ for ${ }^{1} \mathrm{H}$ $\mathrm{NMR}$ and to the central line of $\mathrm{CDCl}_{3}(77.0 \mathrm{ppm})$ for ${ }^{13} \mathrm{C} \mathrm{NMR} .{ }^{1} \mathrm{H}$ and ${ }^{13} \mathrm{C}$ NMR spectra are reported and not described due to the presence of the signals of several rotameric isomers. ${ }^{1} \mathrm{H}$ NMR spectra were recorded also at $60{ }^{\circ} \mathrm{C} .{ }^{13} \mathrm{C}$ NMR spectra were acquired on a broad band decoupled mode. Mass spectra were recorded on a micromass LCT spectrometer using electrospray $\left(\mathrm{ES}^{+}\right)$ionisation techniques. Flash column chromatography (FC) was carried out using the Merck silica gel 60 (230-400 mesh). Optical rotations were measured on a Perkin-Elmer 241 polarimeter. The enantiomeric excess (ee) of the products was determined by chiral stationary phase HPLC (Daicel Chiralpak AD or Daciel Chiralcel OJ/OD columns). Racemic samples were obtained using an equimolar mixture of (S)-(-)-2,2'-isopropylidene-bis(4-phenyl-2-oxazoline) and $(R)-(+)-2,2$ '-isopropylidene-bis(4-phenyl-2-oxazoline) as a ligand.

Materials. $\mathrm{CH}_{2} \mathrm{Cl}_{2}$ was distilled from $\mathrm{CaH}_{2}$ prior to use. All commercially available reagents were used as received. $\beta$-Ketophosphonates $\mathbf{1 a - d}, \mathbf{f}^{1} \mathbf{1} \mathbf{e}^{2}$ and $\mathbf{1 g}, \mathbf{h}^{3}$ were prepared according to literature procedures.

\section{General Procedure for the Catalytic Asymmetric Amination of $\beta$-Ketophosphonates}

1a-h: In a flame dried Schlenk tube equipped with a magnetic stirring bar, $\mathrm{Zn}(\mathrm{OTf})_{2}(7.3$ $\mathrm{mg}, 0.02 \mathrm{mmol}$ ) and (S)-(-)-2,2'-isopropylidene-bis(4-phenyl-2-oxazoline) (7.4 mg, 0.022 mmol) were added. The solid mixture was stirred under vacuum for $1 \mathrm{~h}$, and then the Schlenk tube was filled with $\mathrm{N}_{2}$. Dry $\mathrm{CH}_{2} \mathrm{Cl}_{2}(2 \mathrm{~mL})$ was added, and the resulting colourless solution was stirred for $1 \mathrm{~h}$. $\beta$-Ketophosphonates $\mathbf{1 a - h}(0.2 \mathrm{mmol})$ were then added, followed by azodicarboxylates $\mathbf{2 a}$ or $\mathbf{2 b}(0.3 \mathrm{mmol})$. After $48 \mathrm{~h}$ stirring at room temperature, the products $3 \mathbf{a}-\mathbf{i}$ were isolated by $\mathrm{FC}$ on silica gel $\left(\mathrm{CH}_{2} \mathrm{Cl}_{2} / \mathrm{Et}_{2} \mathrm{O}\right.$ mixtures).

\footnotetext{
${ }^{1}$ Truel, I.; Mohamed-Hachi, A.; Aboud-Jaudet, E.; Collignon, N. Synth. Commun. 1997, $27,1165$.

${ }^{2}$ Clark, R. D.; Kozar, L. G.; Heathcock, C. H. Synthesis 1975, 635.

${ }^{3}$ Calogeropoulou, T.; Hammond, G. B.; Wiemer, D. F. J. Org. Chem. 1987, 52, 4185.
} 


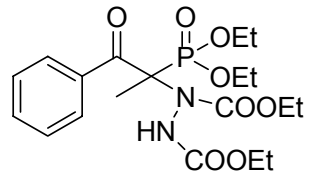

\section{$N, N$ '-Bis(ethoxycarbonyl)-1-hydrazino-1-methyl-2-oxo-2-phenyl-} ethyl phosphonic acid diethyl ester (3a). Following the general procedure, compound 3a was obtained as a colourless oil in $75 \%$

yield. The ee of the product was determined by HPLC using a Daicel Chiralpak AD column $\left(n\right.$-hexane $/ i-\mathrm{PrOH}=90 / 10$, flow rate $1.0 \mathrm{~mL} / \mathrm{min} \tau_{\text {major }}=16.1 \mathrm{~min} ; \tau_{\text {minor }}=9.5$ min); ${ }^{1} \mathrm{H}$ NMR $\left(\mathrm{CDCl}_{3}\right)$ see below; ${ }^{13} \mathrm{C}$ NMR $\left(\mathrm{CDCl}_{3}\right)$ see below; HRMS $\mathrm{C}_{19} \mathrm{H}_{29} \mathrm{~N}_{2} \mathrm{O}_{8} \mathrm{P}$ $[\mathrm{M}+\mathrm{Na}]^{+}$calculated: 467.1554 , found: $467.1554 ;[\alpha]^{\mathrm{rt}}{ }_{\mathrm{D}}=-103\left(c=0.72\right.$ in $\left.\mathrm{CHCl}_{3}\right), 92 \%$ ee.

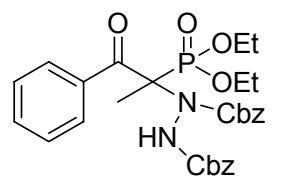

\section{$N, N$ '-Bis(benzyloxycarbonyl)-1-hydrazino-1-methyl-2-oxo-2-phenyl- ethyl phosphonic acid diethyl ester (3b). Following the general procedure, compound $\mathbf{3 b}$ was obtained as a colourless oil in $85 \%$ yield.} The ee of the product was determined by HPLC using a Daicel Chiralpak AD column ( $n$ hexane $/ i-\mathrm{PrOH}=85 / 15$, flow rate $\left.1.0 \mathrm{~mL} / \mathrm{min} \tau_{\text {major }}=21.3 \mathrm{~min} ; \tau_{\text {minor }}=10.9 \mathrm{~min}\right) ;{ }^{1} \mathrm{H}$ NMR $\left(\mathrm{CDCl}_{3}\right)$ see below; ${ }^{13} \mathrm{C}$ NMR $\left(\mathrm{CDCl}_{3}\right)$ see below; HRMS $\mathrm{C}_{29} \mathrm{H}_{33} \mathrm{~N}_{2} \mathrm{O}_{8} \mathrm{P}[\mathrm{M}+\mathrm{Na}]^{+}$ calculated: 591.1867, found: 591.1899; $[\alpha]_{\mathrm{D}}^{\mathrm{rt}}=-82\left(c=0.44\right.$ in $\left.\mathrm{CHCl}_{3}\right), 92 \%$ ee.

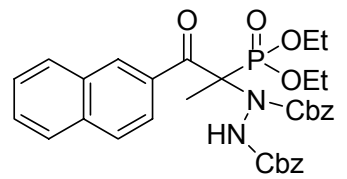

$N, N$ '-Bis(benzyloxycarbonyl)-1-hydrazino-1-methyl-2-naphtalen 2-yl-2-oxo-ethyl phosphonic acid diethyl ester (3c). Following the general procedure, compound $\mathbf{3 c}$ was obtained as a white solid in 93\% yield. The ee of the product was determined by HPLC using a Daicel Chiralpak AD column $\left(n\right.$-hexane $/ i-\mathrm{PrOH}=85 / 15$, flow rate $1.0 \mathrm{~mL} / \mathrm{min} \tau_{\text {major }}=33.1 \mathrm{~min} ; \tau_{\text {minor }}=13.2$ min); m.p. $=39-42{ }^{\circ} \mathrm{C} ;{ }^{1} \mathrm{H}$ NMR $\left(\mathrm{CDCl}_{3}\right)$ see below; ${ }^{13} \mathrm{C}$ NMR $\left(\mathrm{CDCl}_{3}\right)$ see below; HRMS $\mathrm{C}_{33} \mathrm{H}_{35} \mathrm{~N}_{2} \mathrm{O}_{8} \mathrm{P}[\mathrm{M}+\mathrm{Na}]^{+}$calculated: 641.2023 , found: $641.2321 ;[\alpha]^{\mathrm{rt}}=-75(c=$ 0.36 in $\left.\mathrm{CHCl}_{3}\right), 92 \%$ ee.

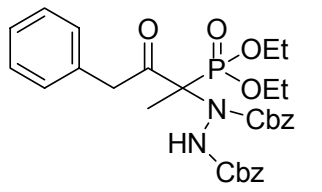

$N, N$ '-Bis(benzyloxycarbonyl)-1-hydrazino-1-methyl-2-oxo-3phenyl-propyl phosphonic acid diethyl ester (3d). Following the general procedure, compound $\mathbf{3 d}$ was obtained as a colourless oil in $60 \%$ yield. The ee of the product was determined by HPLC using a Daicel Chiralcel OD column $\left(n\right.$-hexane $/ i-\mathrm{PrOH}=90 / 10$, flow rate $1.0 \mathrm{~mL} / \mathrm{min} \tau_{\text {major }}=12.8 \mathrm{~min} ; \tau_{\text {minor }}=18.0$ 
min); ${ }^{1} \mathrm{H}$ NMR $\left(\mathrm{CDCl}_{3}\right)$ see below; ${ }^{13} \mathrm{C}$ NMR $\left(\mathrm{CDCl}_{3}\right)$ see below; HRMS $\mathrm{C}_{30} \mathrm{H}_{35} \mathrm{~N}_{2} \mathrm{O}_{8} \mathrm{P}$ $[\mathrm{M}+\mathrm{Na}]^{+}$calculated: 605.2023 , found: $605.2019 ;[\alpha]_{\mathrm{D}}^{\mathrm{rt}}=-25\left(c=0.50\right.$ in $\left.\mathrm{CHCl}_{3}\right), 95 \%$ ee.

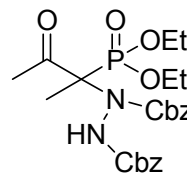

$N, N$ '-Bis(benzyloxycarbonyl)-1-hydrazino-1-methyl-2-oxo-ethyl phosphonic acid diethyl ester (3e). Following the general procedure, compound 3e was obtained as a white solid in $75 \%$ yield. The ee of the product was determined on the corresponding oxazolidinone (vide infra); m.p. $=85-88$ ${ }^{\circ} \mathrm{C}$; ${ }^{1} \mathrm{H}$ NMR $\left(\mathrm{CDCl}_{3}\right)$ see below; ${ }^{13} \mathrm{C}$ NMR $\left(\mathrm{CDCl}_{3}\right)$ see below; HRMS $\mathrm{C}_{24} \mathrm{H}_{31} \mathrm{~N}_{2} \mathrm{O}_{8} \mathrm{P}$ $[\mathrm{M}+\mathrm{Na}]^{+}$calculated: 529.1710 , found: $529.1738 ;[\alpha]^{\mathrm{rt}}=-29\left(c=0.37\right.$ in $\left.\mathrm{CHCl}_{3}\right), 85 \%$ ee.

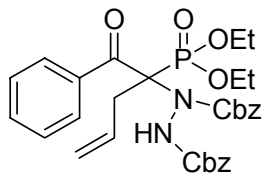

$N, N$ '-Bis(benzyloxycarbonyl)-1-hydrazino-2-oxo-2-phenylethyl-1prop-2-en phosphonic acid diethyl ester (3f). Following the general procedure and prolonging the reaction time to $96 \mathrm{~h}$ compound $3 \mathbf{f}$ was obtained as a colourless oil in $85 \%$ yield. The ee of the product was determined on the corresponding oxazolidinone (vide infra); ${ }^{1} \mathrm{H}$ NMR $\left(\mathrm{CDCl}_{3}\right)$ see below; ${ }^{13} \mathrm{C}$ NMR $\left(\mathrm{CDCl}_{3}\right)$ see below; HRMS $\mathrm{C}_{31} \mathrm{H}_{35} \mathrm{~N}_{2} \mathrm{O}_{8} \mathrm{P}[\mathrm{M}+\mathrm{Na}]^{+}$calculated: 617.2023, found: $617.2020 ;[\alpha]^{\mathrm{rt}}=-56\left(c=0.35\right.$ in $\left.\mathrm{CHCl}_{3}\right), 97 \%$ ee.

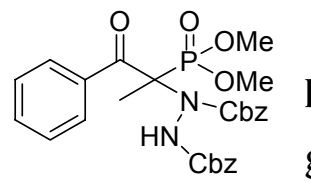

$N, N$ '-Bis(benzyloxycarbonyl)-1-hydrazino-1-methyl-2-oxo-2phenyl-ethyl phosphonic acid dimethyl ester (3g). Following the general procedure, compound $\mathbf{3 g}$ was obtained as a white solid in $97 \%$ yield, after chromatography on silica gel. The ee of the product was determined by HPLC using a Daicel Chiralcel OJ column ( $n$-hexane $/ i-\mathrm{PrOH}=85 / 15$, flow rate $1.0 \mathrm{~mL} / \mathrm{min}$ $\left.\tau_{\text {major }}=27.2 \mathrm{~min} ; \tau_{\text {minor }}=13.8 \mathrm{~min}\right)$; m.p. $=38-40{ }^{\circ} \mathrm{C} ;{ }^{1} \mathrm{H}$ NMR $\left(\mathrm{CDCl}_{3}\right)$ see below; ${ }^{13} \mathrm{C}$ NMR $\left(\mathrm{CDCl}_{3}\right)$ see below; HRMS $\mathrm{C}_{29} \mathrm{H}_{33} \mathrm{~N}_{2} \mathrm{O}_{8} \mathrm{P}[\mathrm{M}+\mathrm{Na}]^{+}$calculated: 591.1867, found: $591.1899 ;[\alpha]_{\mathrm{D}}^{\mathrm{rt}}=-106\left(c=0.45\right.$ in $\left.\mathrm{CHCl}_{3}\right), 95 \%$ ee. 


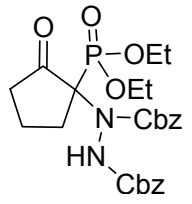
$N, N$ '-Bis(benzyloxycarbonyl)-1-hydrazino-2-oxo-cyclopentyl phosphonic acid diethyl ester (3h). Following the general procedure, compound $3 \mathbf{h}$ was obtained as colourless oil in $98 \%$ yield. The ee of the product was determined by HPLC using a Daicel Chiralcel OD column ( $n$-hexane $i-\mathrm{PrOH}=90 / 10$, flow rate $\left.1.0 \mathrm{~mL} / \mathrm{min} \tau_{\text {major }}=8.9 \mathrm{~min} ; \tau_{\text {minor }}=20.6 \mathrm{~min}\right) ;{ }^{1} \mathrm{H}$ NMR $\left(\mathrm{CDCl}_{3}\right)$ see below; ${ }^{13} \mathrm{C}$ NMR $\left(\mathrm{CDCl}_{3}\right)$ see below; HRMS $\mathrm{C}_{25} \mathrm{H}_{31} \mathrm{~N}_{2} \mathrm{O}_{8} \mathrm{P}[\mathrm{M}+\mathrm{Na}]^{+}$calculated: 541.1710, found: $541.1712 ;[\alpha]_{D}^{\mathrm{rt}}=+41\left(c=0.22\right.$ in $\left.\mathrm{CHCl}_{3}\right), 95 \%$ ee.

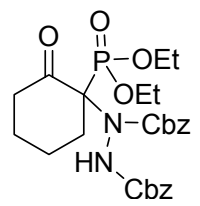

$N, N^{\prime}$-Bis(benzyloxycarbonyl)-1-hydrazino-2-oxo-cyclohexyl phosphonic
acid diethyl ester (3i). Following the general procedure, compound $3 \mathbf{i}$ was
obtained as colourless oil in $98 \%$ yield. The ee of the product was determined by HPLC using a Daicel Chiralcel OD column ( $n$-hexane $/ i-\operatorname{PrOH}=95 / 5$, flow rate $\left.1.0 \mathrm{~mL} / \mathrm{min} \tau_{\text {major }}=28.4 \mathrm{~min} ; \tau_{\text {minor }}=35.7 \mathrm{~min}\right) ;{ }^{1} \mathrm{H} \mathrm{NMR}\left(\mathrm{CDCl}_{3}\right)$ see below; ${ }^{13} \mathrm{C}$ NMR $\left(\mathrm{CDCl}_{3}\right)$ see below; HRMS $\mathrm{C}_{26} \mathrm{H}_{33} \mathrm{~N}_{2} \mathrm{O}_{8} \mathrm{P}[\mathrm{M}+\mathrm{Na}]^{+}$calculated: 555.1867, found: $555.1887 ;[\alpha]_{D}^{\mathrm{rt}}=-27\left(c=0.61\right.$ in $\left.\mathrm{CHCl}_{3}\right), 94 \%$ ee.

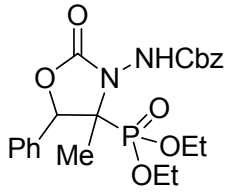

(3-Benzyloxycarbonylamino-4-methyl-2-oxo-5-phenyl-oxazolidin-4yl)-phosphonic acid diethyl ester (4). Compound 3b (461 mg, 0.33 mmol) was dissolved in $\operatorname{dry} \mathrm{Et}_{2} \mathrm{O}(3 \mathrm{~mL})$ and the resulting solution cooled to $-24{ }^{\circ} \mathrm{C} . \mathrm{LiBH}_{4}(36 \mathrm{mg}, 1.65 \mathrm{mmol})$ was added in small portions and the mixture was stirred for $3 \mathrm{~h}$ at $-24{ }^{\circ} \mathrm{C}$. The reaction mixture was then warmed to room temperature and $5 \mathrm{~mL}$ of $\mathrm{H}_{2} \mathrm{O}$ were added. The aqueous suspension was extracted three times with EtOAc. The organic phase was washed with brine, dried on $\mathrm{Na}_{2} \mathrm{SO}_{4}$, filtered and concentrated in vacuo. The crude product was purified by $\mathrm{FC}$ on silica gel $\left(\mathrm{CH}_{2} \mathrm{Cl}_{2} / \mathrm{Et}_{2} \mathrm{O}\right.$ mixtures) to give compound $4(252 \mathrm{mg})$ as a colorless oil in $67 \%$ yield and as a single diastereoisomer. ${ }^{1} \mathrm{H}$ NMR $\left(\mathrm{CDCl}_{3}\right)$ see below; HRMS $\mathrm{C}_{22} \mathrm{H}_{27} \mathrm{~N}_{2} \mathrm{O}_{7} \mathrm{P}[\mathrm{M}+\mathrm{Na}]^{+}$; calculated: 485.1454 found: 485.1455 .

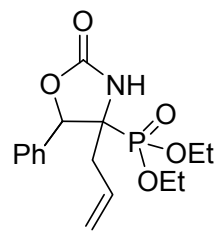

(4-Allyl-3-benzyloxycarbonylamino-2-oxo-5-phenyl-oxazolidin-4-yl)phosphonic acid diethyl ester. Following the procedure used for compound $\mathbf{4}$, the title compound was obtained in $29 \%$ yield as pale yellow 
oil and as a single diastereoisomer. The ee of the product was determined by HPLC using a Daicel Chiralpak AD column ( $n$-hexane $/ i-\mathrm{PrOH}=90 / 10$, flow rate $1.0 \mathrm{~mL} / \mathrm{min} \tau_{\text {major }}=$ $\left.24.2 \mathrm{~min} ; \tau_{\text {minor }}=31.5 \mathrm{~min}\right) .{ }^{1} \mathrm{H} \mathrm{NMR}\left(\mathrm{CDCl}_{3}\right)$ see below; HRMS $\mathrm{C}_{24} \mathrm{H}_{29} \mathrm{~N}_{2} \mathrm{O}_{7} \mathrm{P}[\mathrm{M}+\mathrm{Na}]^{+}$ calculated: 511.1610, found: $511.1614,97 \%$ ee.

(4-Methyl-2-oxo-5-phenyl-oxazolidin-4-yl)-phosphonic acid diethyl
ester. (5). To a solution of compound $3 \mathbf{b}(69 \mathrm{mg}, \mathrm{mmol})$ in $\mathrm{MeOH}(4 \mathrm{~mL})$ and the mixture was stirred for $2 \mathrm{~h}$ at room temperature. The reaction mixture was filtered through a short pad of Celite and the pad washed with $\mathrm{MeOH}$. After removal of the solvent in vacuo, the oily residue was dissolved in $\mathrm{AcOH}(6 \mathrm{~mL})$ and $1 \mathrm{M} \mathrm{HCl}(2 \mathrm{~mL})$ was added, followed by $\mathrm{NaNO}_{2}(40 \mathrm{mg})$ dissolved in $\mathrm{H}_{2} \mathrm{O}(1 \mathrm{~mL})$. The mixture was refluxed at $110{ }^{\circ} \mathrm{C}$ for $90 \mathrm{~min}$, then cooled to room temperature. $30 \mathrm{~mL}$ sat. $\mathrm{NaHCO}_{3}$ and solid $\mathrm{Na}_{2} \mathrm{CO}_{3}$ were then added to adjust the $\mathrm{pH}$ value to 7 . The water phase was extracted three times by EtOAc and the combined organic phases were washed three times by sat. $\mathrm{NaHCO}_{3}, \mathrm{H}_{2} \mathrm{O}$ and brine subsequently and dried with $\mathrm{Na}_{2} \mathrm{SO}_{4}$. After filtration and removal of the solvents, compound $5(30 \mathrm{mg}$ ) was obtained as a pale yellow oil in $60 \%$ yield. The ee of the product was determined by HPLC using a Daicel Chiralpak AD column ( $n$-hexane $/ i$-PrOH $=90 / 10$, flow rate $1.0 \mathrm{~mL} / \mathrm{min} \tau_{\text {minor }}=20.8 \mathrm{~min}$; $\tau_{\text {major }}=22.9$ min). ${ }^{1} \mathrm{H} \mathrm{NMR}\left(\mathrm{CDCl}_{3}\right) \delta$ 7.45-7.32 (m, 5H), 6.01 (br s, $\left.1 \mathrm{H}\right), 5.38(\mathrm{~d}, J=28.8 \mathrm{~Hz}, 1 \mathrm{H})$, 4.02-3.65 (m, 4H), $1.74(\mathrm{~d}, J=13.2 \mathrm{~Hz}, 3 \mathrm{H}), 1.20(\mathrm{t}, J=7.2 \mathrm{~Hz}, 3 \mathrm{H}), 1.04$ (t, $J=7.2 \mathrm{~Hz}$, $3 \mathrm{H}) ;{ }^{13} \mathrm{C}$ NMR $\delta 157.4,133.0,128.9,128.0,126.8,86.1,63.8,63.7,62.6,62.5,22.1$, 22.0, 16.4, 16.0; HRMS $\mathrm{C}_{14} \mathrm{H}_{20} \mathrm{NO}_{5} \mathrm{P}[\mathrm{M}+\mathrm{Na}]^{+}$; calculated: 336.0977 found: 336.0978 ; $[\alpha]^{\mathrm{rt}} \mathrm{D}=-34\left(c=2.3\right.$ in $\left.\mathrm{CHCl}_{3}\right), 92 \%$ ee. 
Some representative Results from the Screening of Different Catalysts and Solvents and Additives for the Catalytic Amination Reaction of 1a with 2a and 2b.

\begin{tabular}{|c|c|c|c|c|c|c|}
\hline & O & $\begin{array}{r}\mathrm{R}^{1} \mathrm{O} \\
+\quad\end{array}$ & $\begin{array}{rl} & \\
\mathrm{CO}_{2} \mathrm{R}^{1} & \mathrm{C} \\
= & \mathrm{Et} \\
= & \mathrm{Bn}\end{array}$ & $\frac{\text { it. }(10 \mathrm{~mol} \%)}{\text { r.t., } 20 \mathrm{~h}}$ & $\begin{array}{l}\text { a: } \mathrm{R}=\mathrm{Et} \\
\text { b: } \mathrm{R}=\mathrm{Bn}\end{array}$ & \\
\hline Entry & Catalyst & $\begin{array}{l}\text { Azodicar- } \\
\text { boxylate }\end{array}$ & Solvent & Additive & Conversion $^{\mathrm{a}}$ & $\mathrm{Ee}^{\mathrm{b}}$ \\
\hline 1 & $\underset{t-\mathrm{Bu}}{\gamma^{\mathrm{N}} \underset{\mathrm{Cu}}{\mathrm{N}}}$ & $2 a$ & $\mathrm{CH}_{2} \mathrm{Cl}_{2}$ & & $<10$ & / \\
\hline 2 & $t-\mathrm{Bu}$ & $2 a$ & $\mathrm{Et}_{2} \mathrm{O}$ & & $<10$ & I \\
\hline 3 & & $2 a$ & $\mathrm{CH}_{2} \mathrm{Cl}_{2}$ & & 50 & 20 \\
\hline 4 & $(\mathrm{OTf})_{2}$ & $2 \mathbf{a}$ & $\mathrm{Et}_{2} \mathrm{O}$ & & 60 & 0 \\
\hline 5 & $(\mathrm{OTf})_{2}$ & $2 a$ & THF & & 20 & 12 \\
\hline 6 & & $2 \mathbf{a}$ & $\mathrm{CH}_{2} \mathrm{Cl}_{2}$ & & 57 & 19 \\
\hline 7 & $(\mathrm{OTt})_{2}$ & $2 \mathbf{a}$ & $\mathrm{CH}_{2} \mathrm{Cl}_{2}$ & & 52 & 0 \\
\hline 8 & & $2 a$ & $\mathrm{CH}_{2} \mathrm{Cl}_{2}$ & $4 \AA ̊ \mathrm{MS}$ & $>90$ & 29 \\
\hline 9 & (ClO & $2 \mathbf{a}$ & $\mathrm{CH}_{2} \mathrm{Cl}_{2}$ & $4 \AA \mathrm{MS}$ & 80 & 91 \\
\hline 10 & $\begin{array}{l}\mathrm{Zn} \\
(\mathrm{OTf})_{2} \\
4 \mathrm{c}\end{array}$ & $2 \mathbf{a}$ & Toluene & & 80 & 79 \\
\hline
\end{tabular}




\begin{tabular}{ccccccc}
\hline 11 & $\mathbf{4 c}$ & $\mathbf{2 a}$ & $\mathrm{THF}$ & 85 & 86 \\
\hline 12 & $\mathbf{4 c}$ & $\mathbf{2 a}$ & $\mathrm{CHCl}_{3}$ & & 66 & 85 \\
\hline 13 & $\mathbf{4 c}$ & $\mathbf{2 b}$ & $\mathrm{CH}_{2} \mathrm{Cl}_{2}$ & $\mathrm{Et}_{3} \mathrm{~N}\left(10 \mathrm{~mol}_{\mathrm{o}}\right)$ & $80^{\mathrm{c}}$ & 92
\end{tabular}

${ }^{a}$ Determined by ${ }^{1}$ H NMR spectroscopy. ${ }^{b}$ Determined by chiral stationary phase HPLC. ${ }^{\mathrm{c}}$ 48h reaction. 


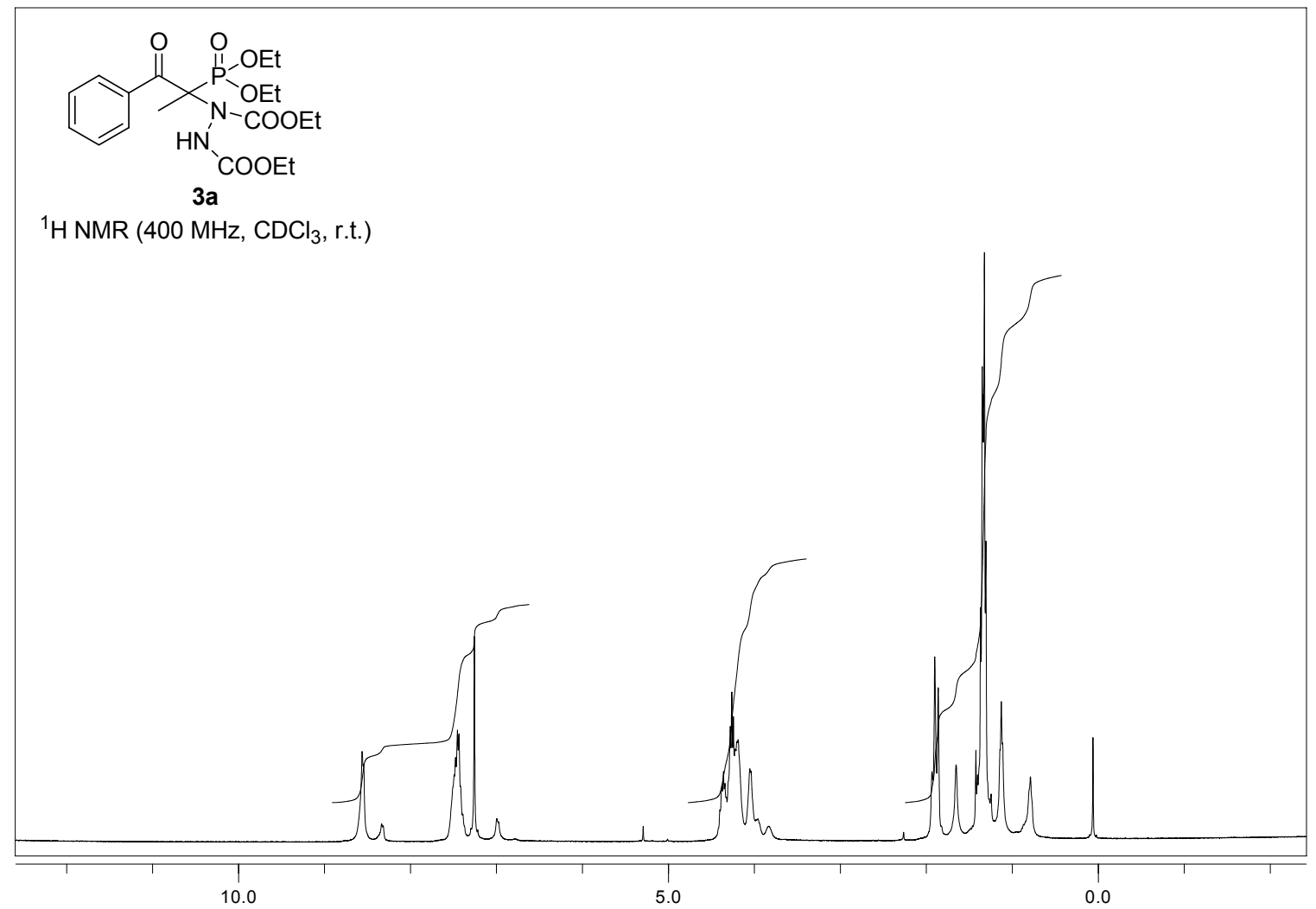

ppm (f1)

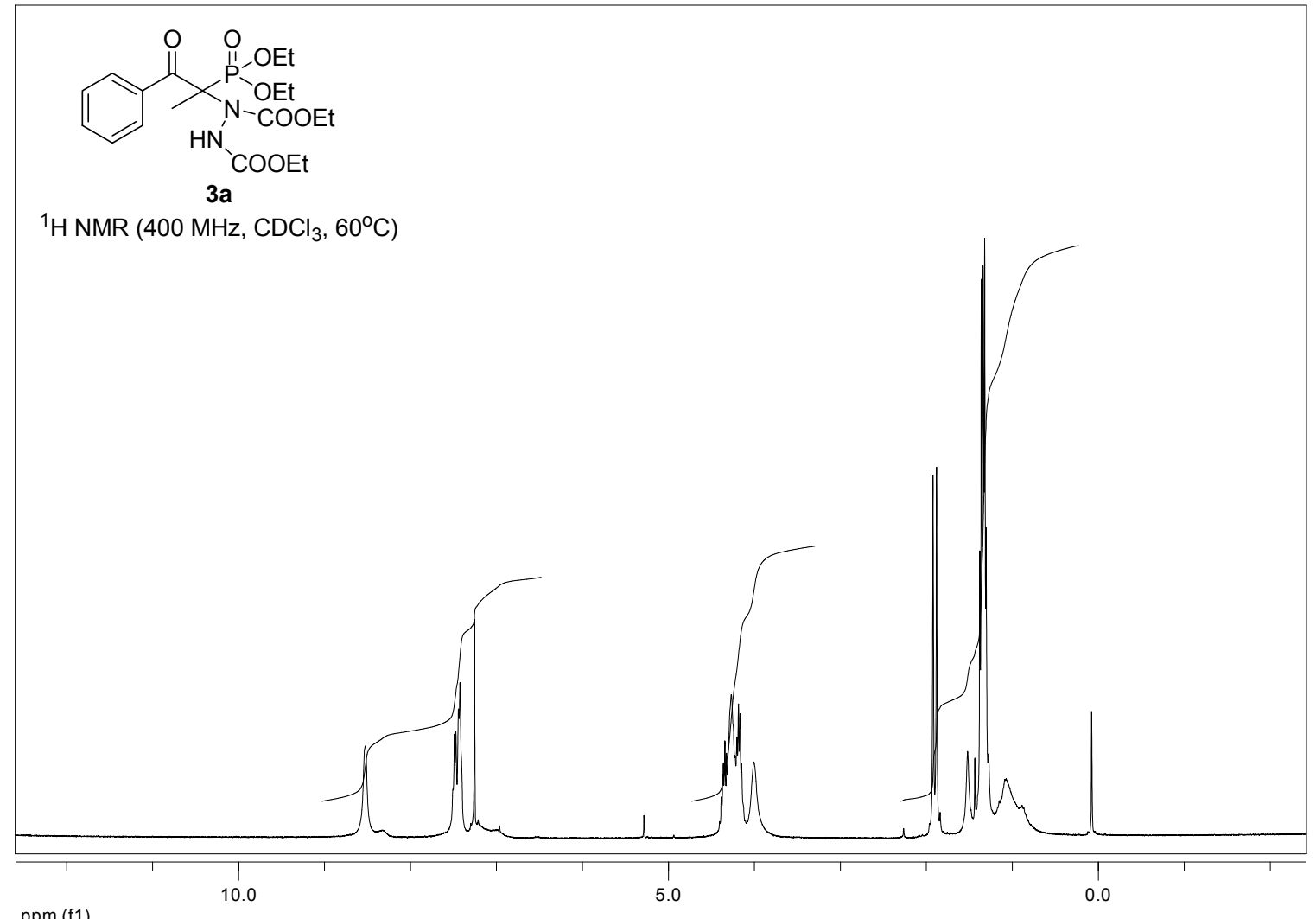

ppm (f1) 


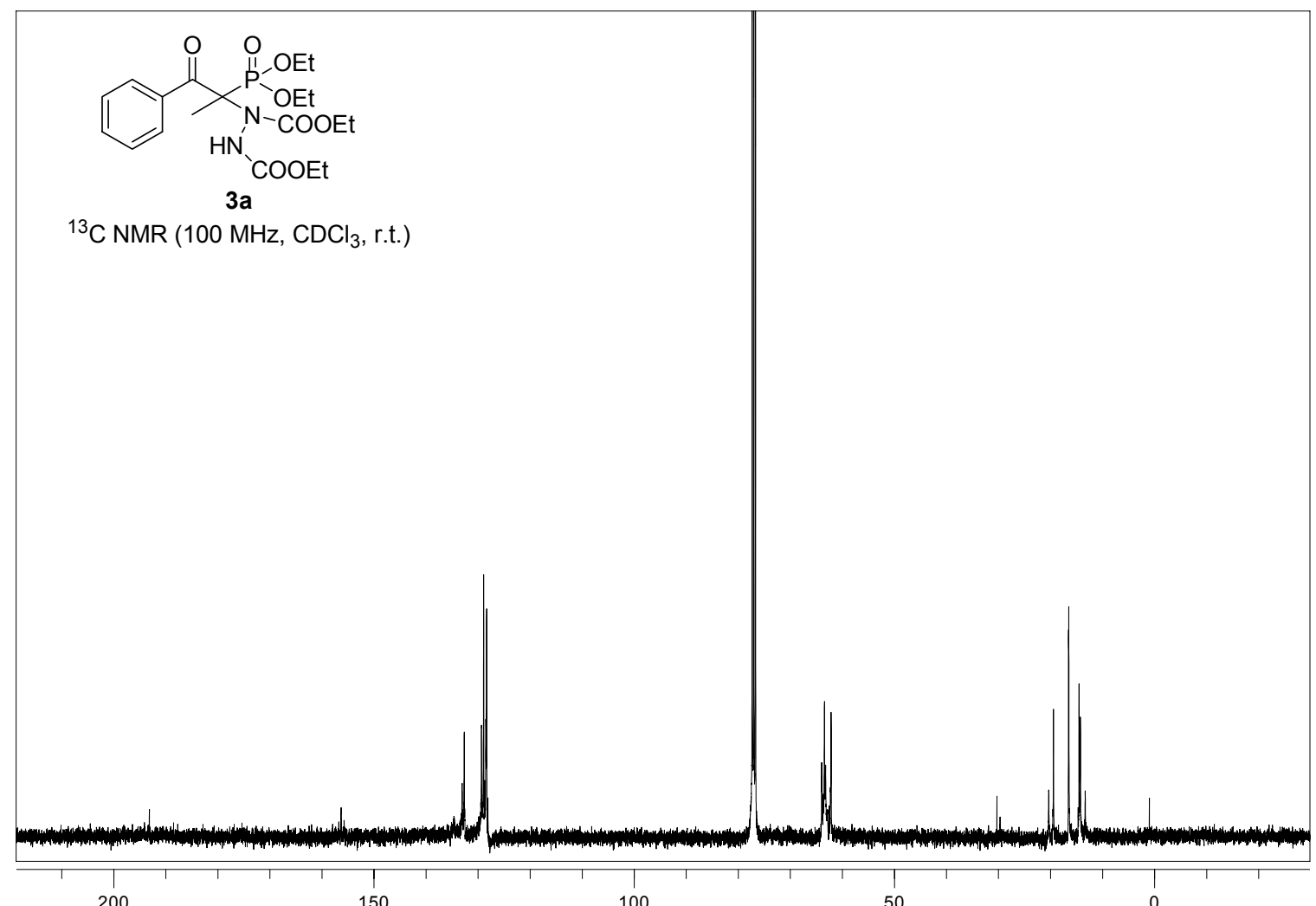

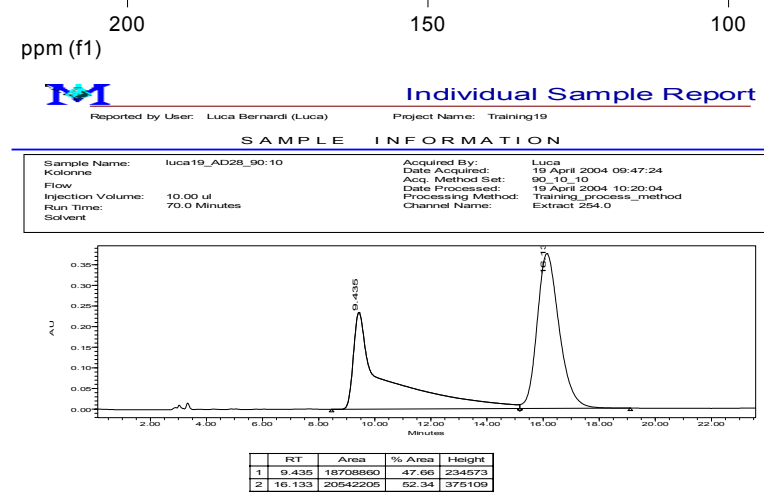

rac-3a

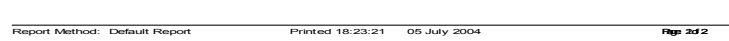

POI Irohidas Sandereport
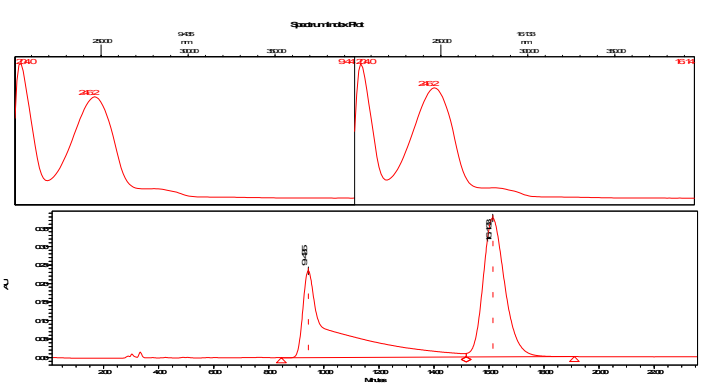

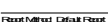
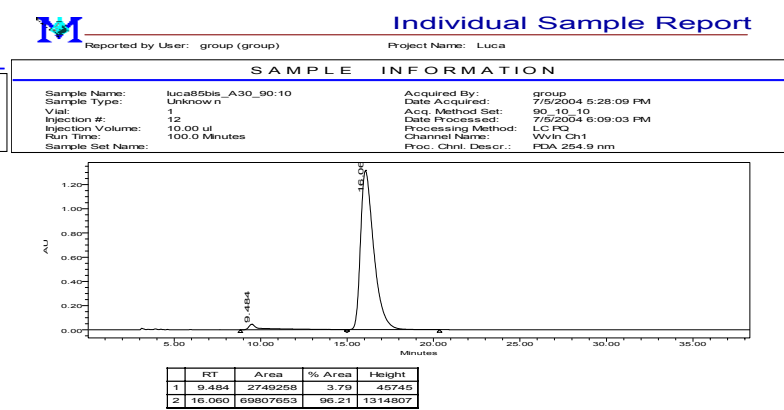

$(-)-3 a$

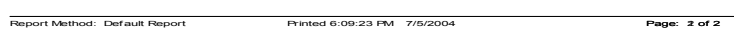

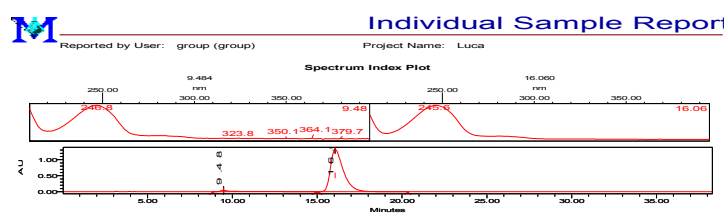

Report Method: Default Report 


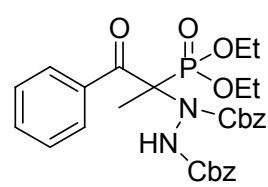

3b

${ }^{1} \mathrm{H}$ NMR (400 MHz, $\mathrm{CDCl}_{3}$, r.t.)

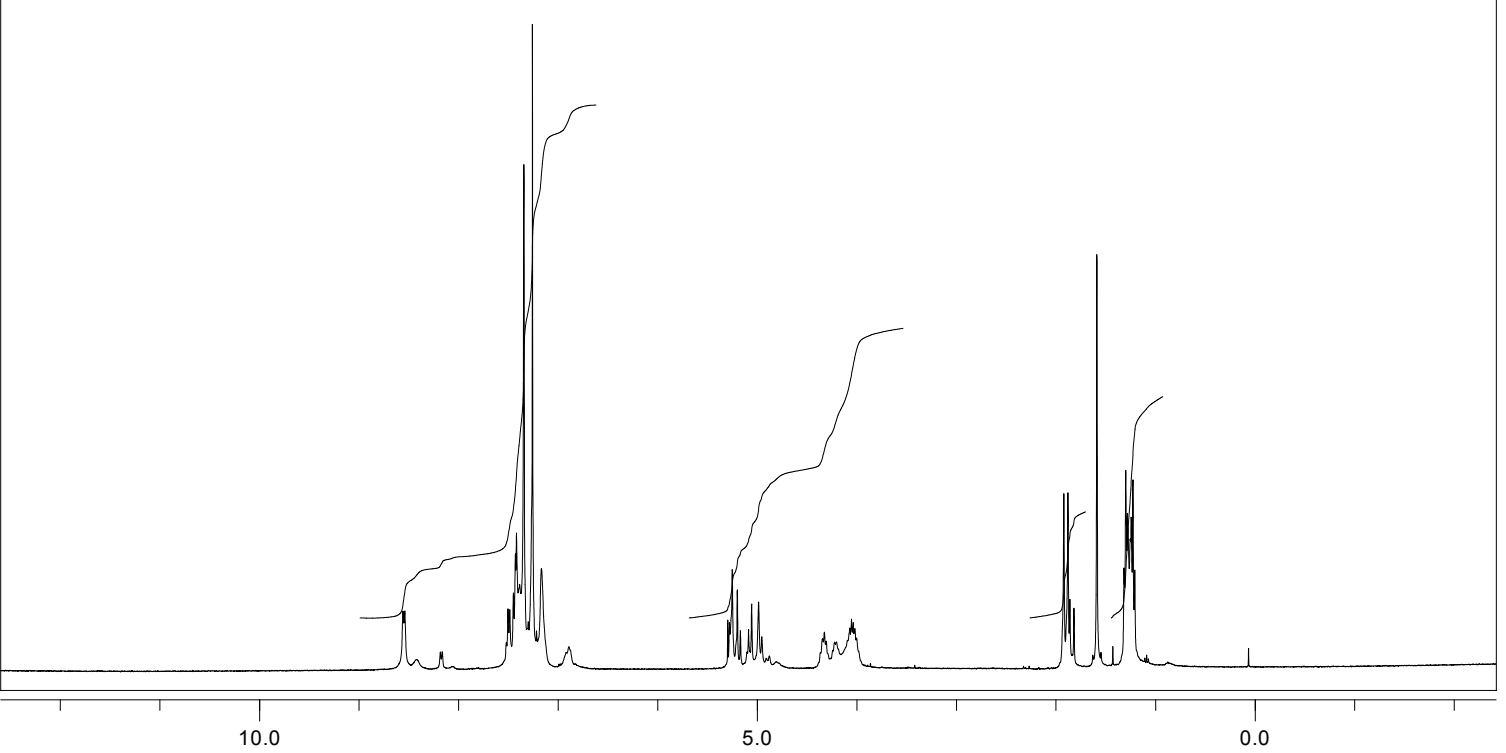

ppm (t1)

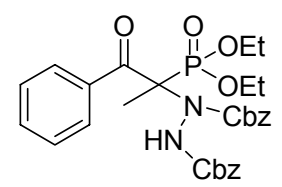

$3 b$

${ }^{1} \mathrm{H}$ NMR $\left(400 \mathrm{MHz}, \mathrm{CDCl}_{3}, 60^{\circ} \mathrm{C}\right)$

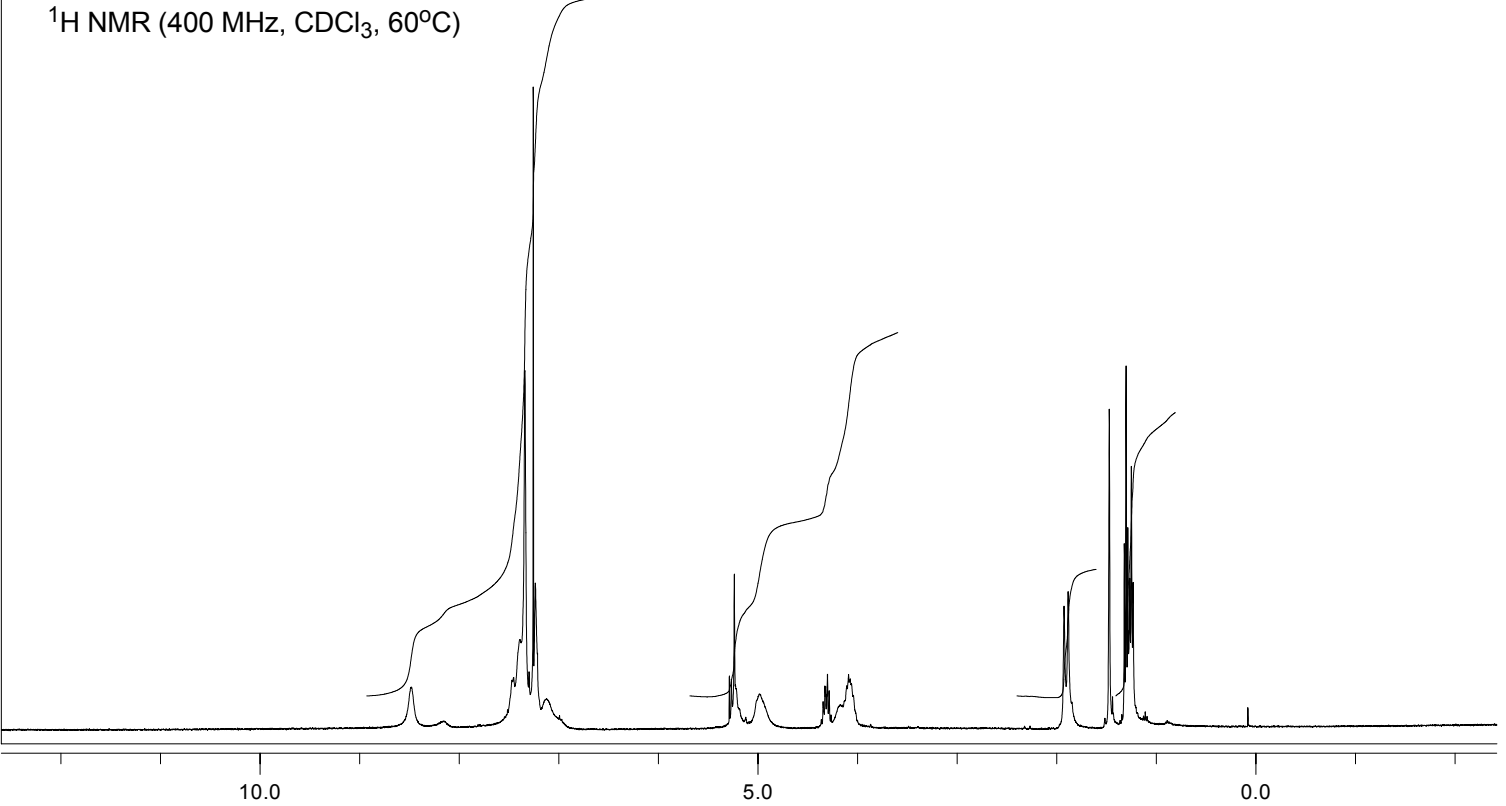

ppm (t1) 


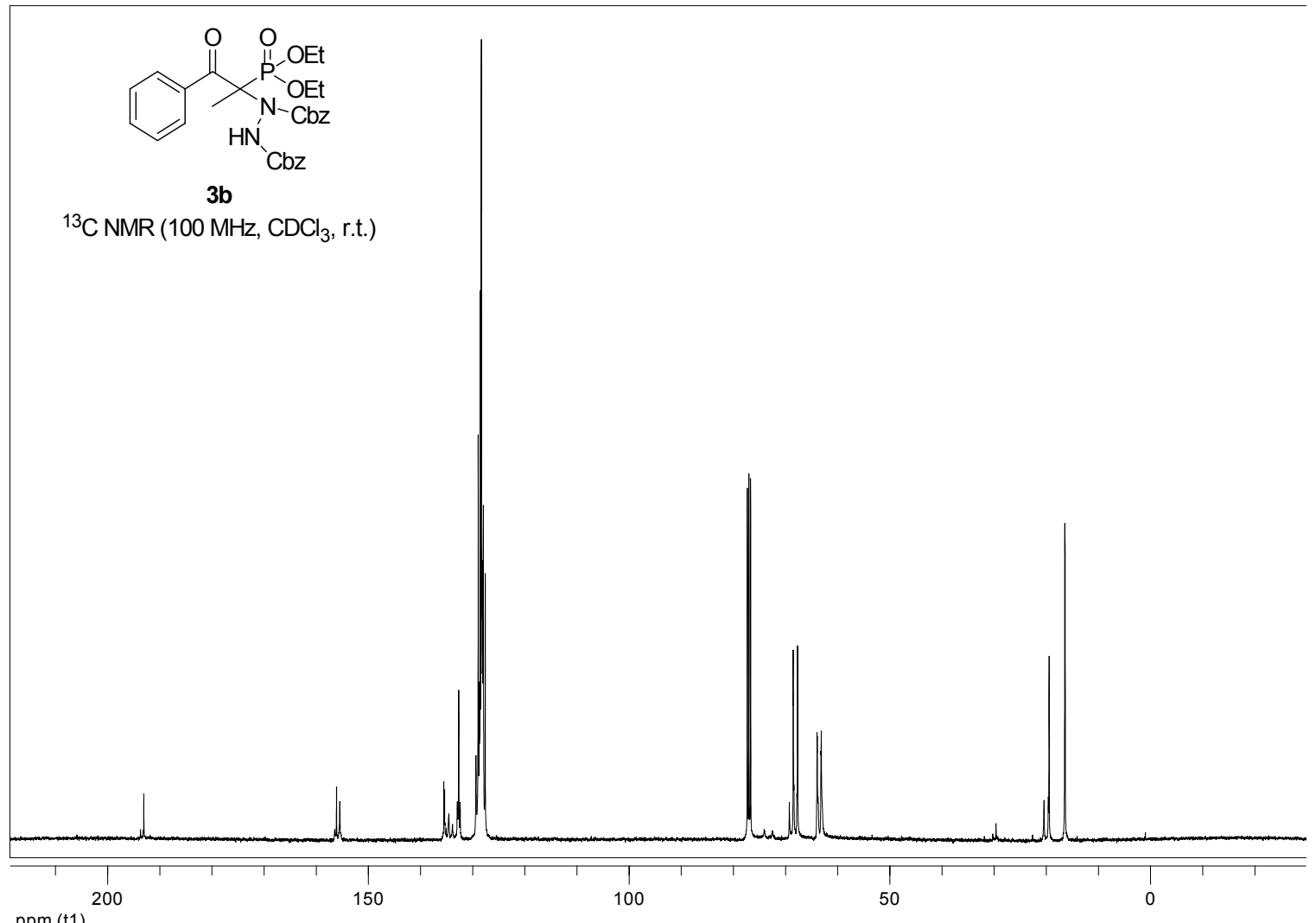

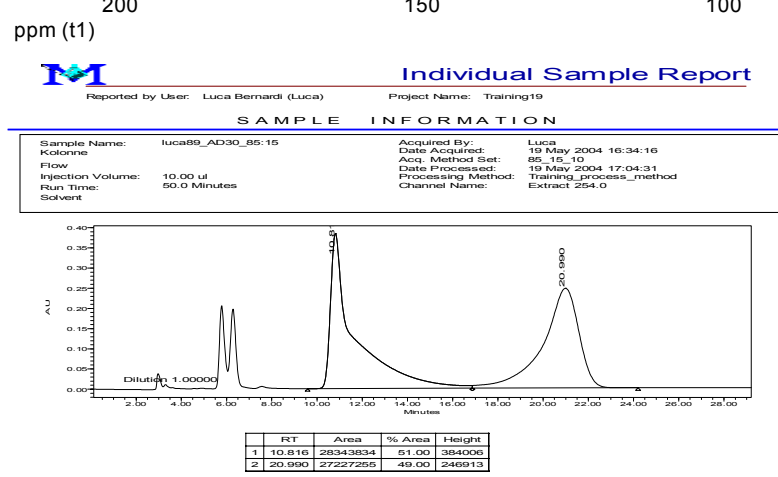

rac-3b

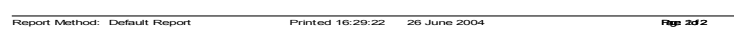

$\mathbf{T}=1$

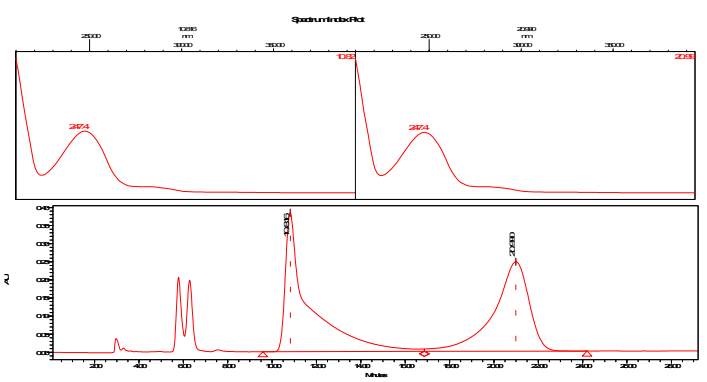

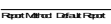
Irolidas Sanderepont

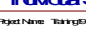

M
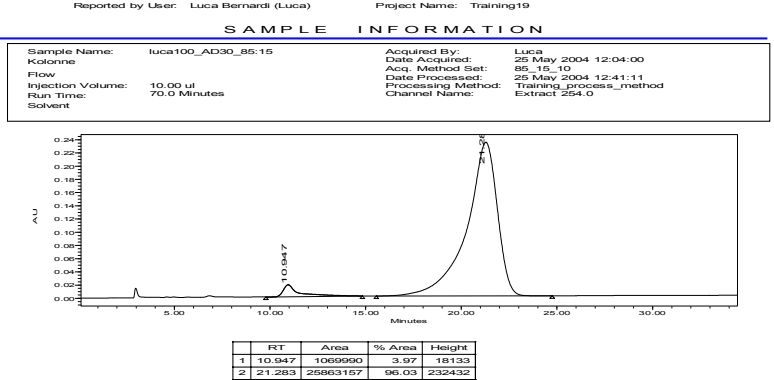

$(-)-3 b$
$\mathbf{I}$

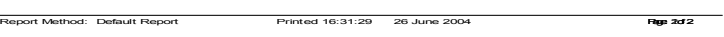

Irohidas Sonple Rant

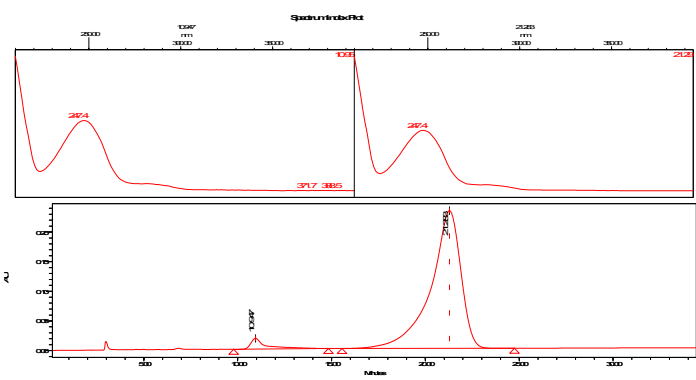

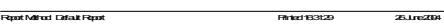




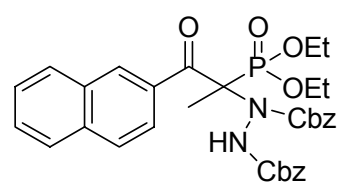

$3 c$

${ }^{1} \mathrm{H}$ NMR (400 MHz, $\mathrm{CDCl}_{3}$, r.t.)
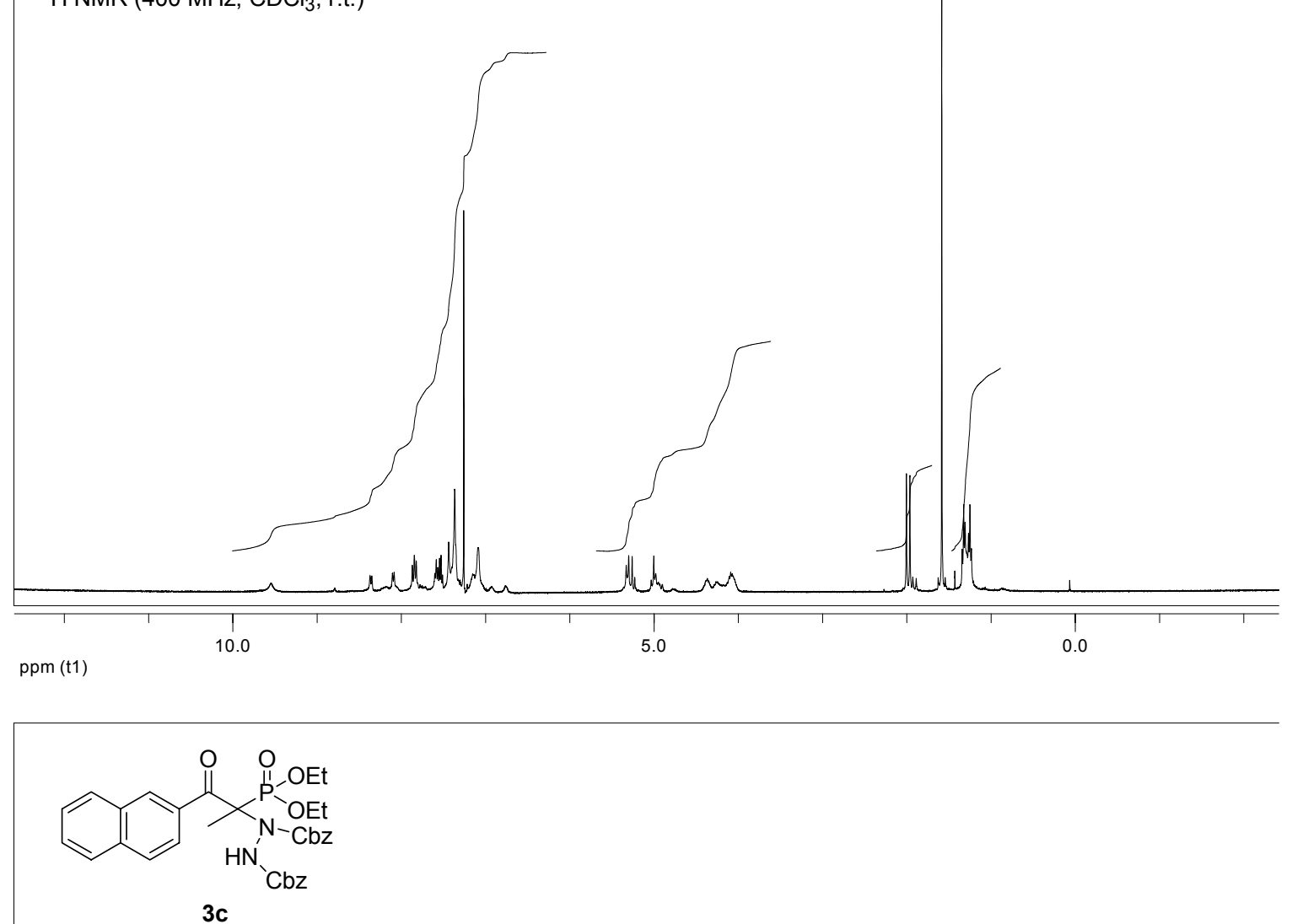

${ }^{1} \mathrm{H}$ NMR $\left(400 \mathrm{MHz}, \mathrm{CDCl}_{3}, 60^{\circ} \mathrm{C}\right)$

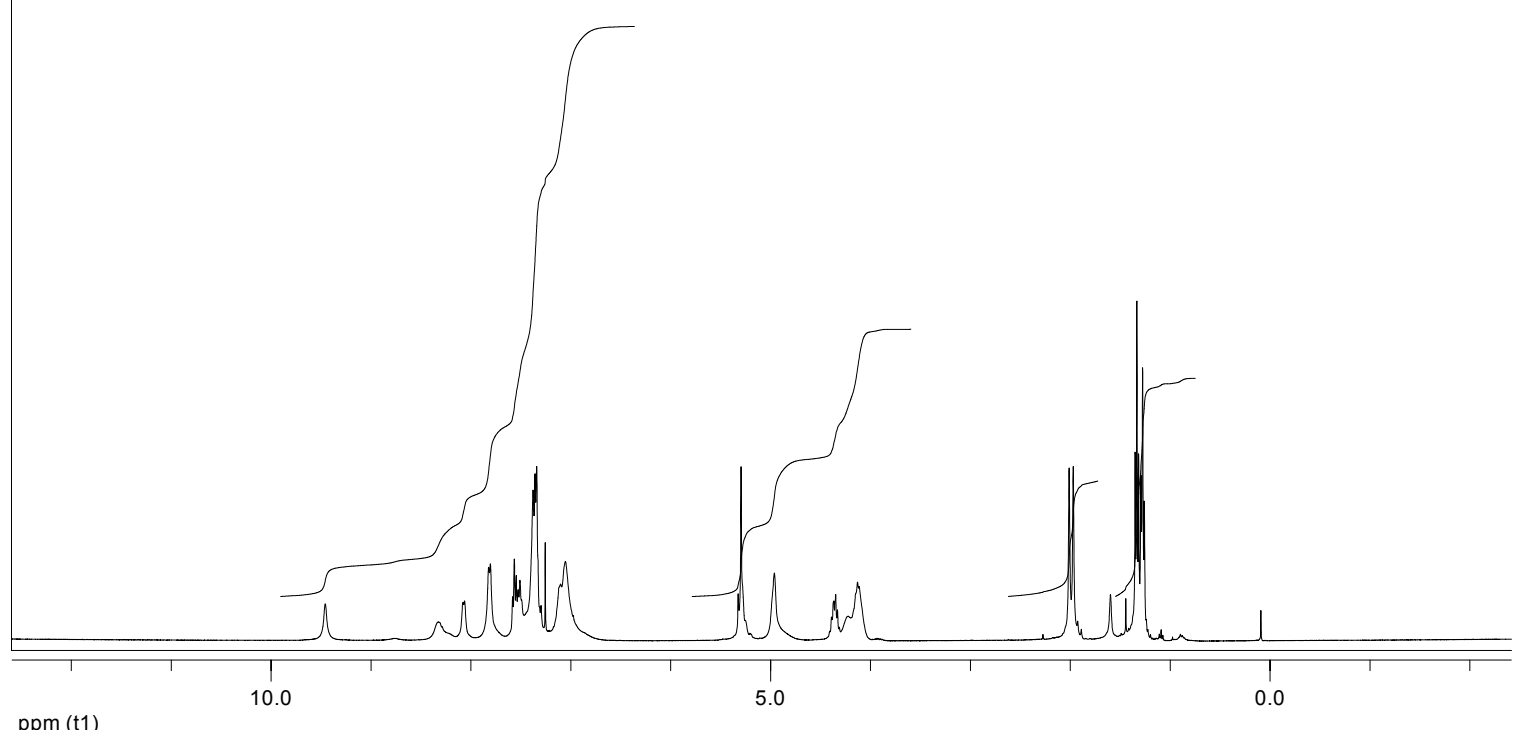



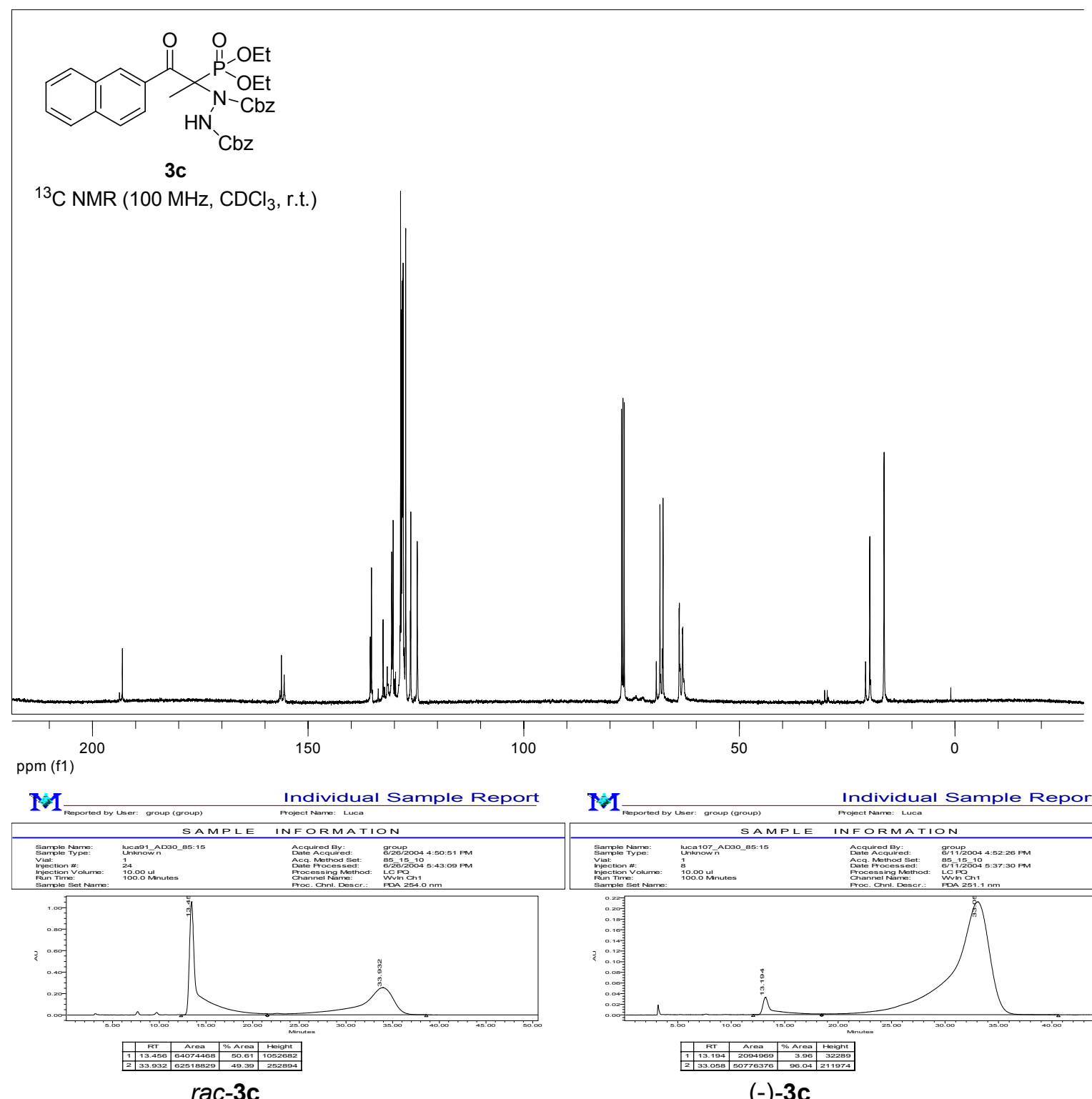

rac-3c

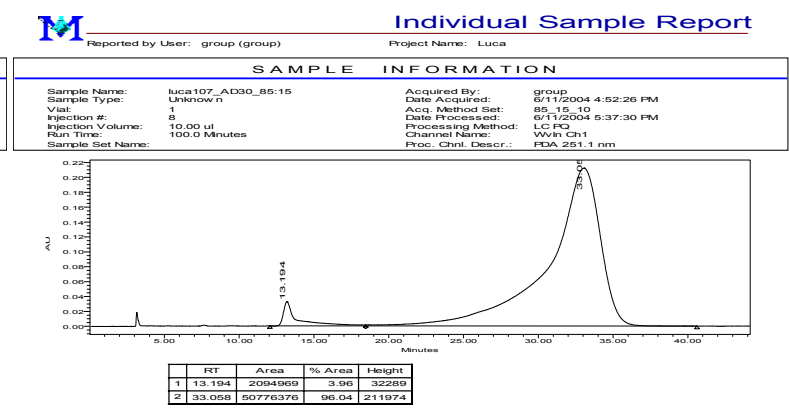

$(-)-3 c$
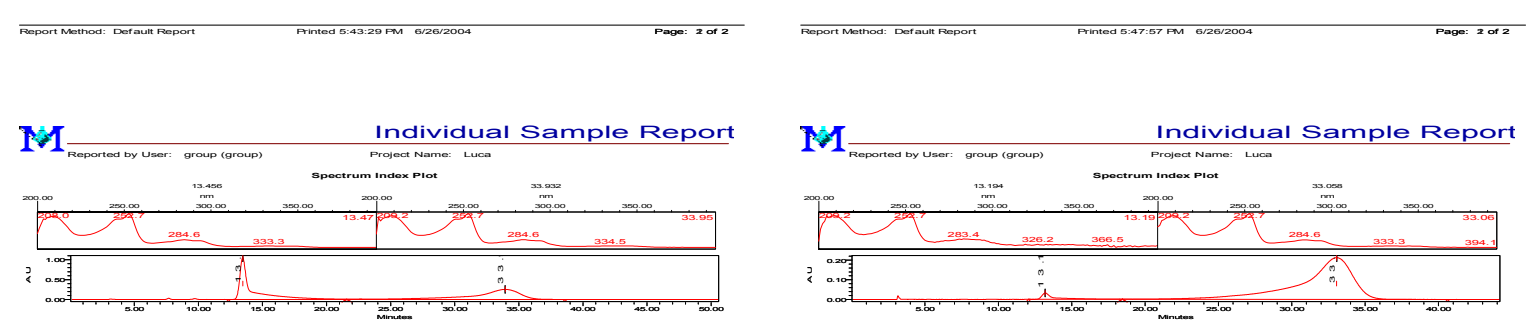


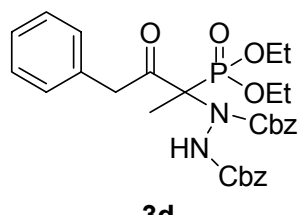

${ }^{1} \mathrm{H}$ NMR (400 MHz, $\mathrm{CDCl}_{3}$, r.t.)

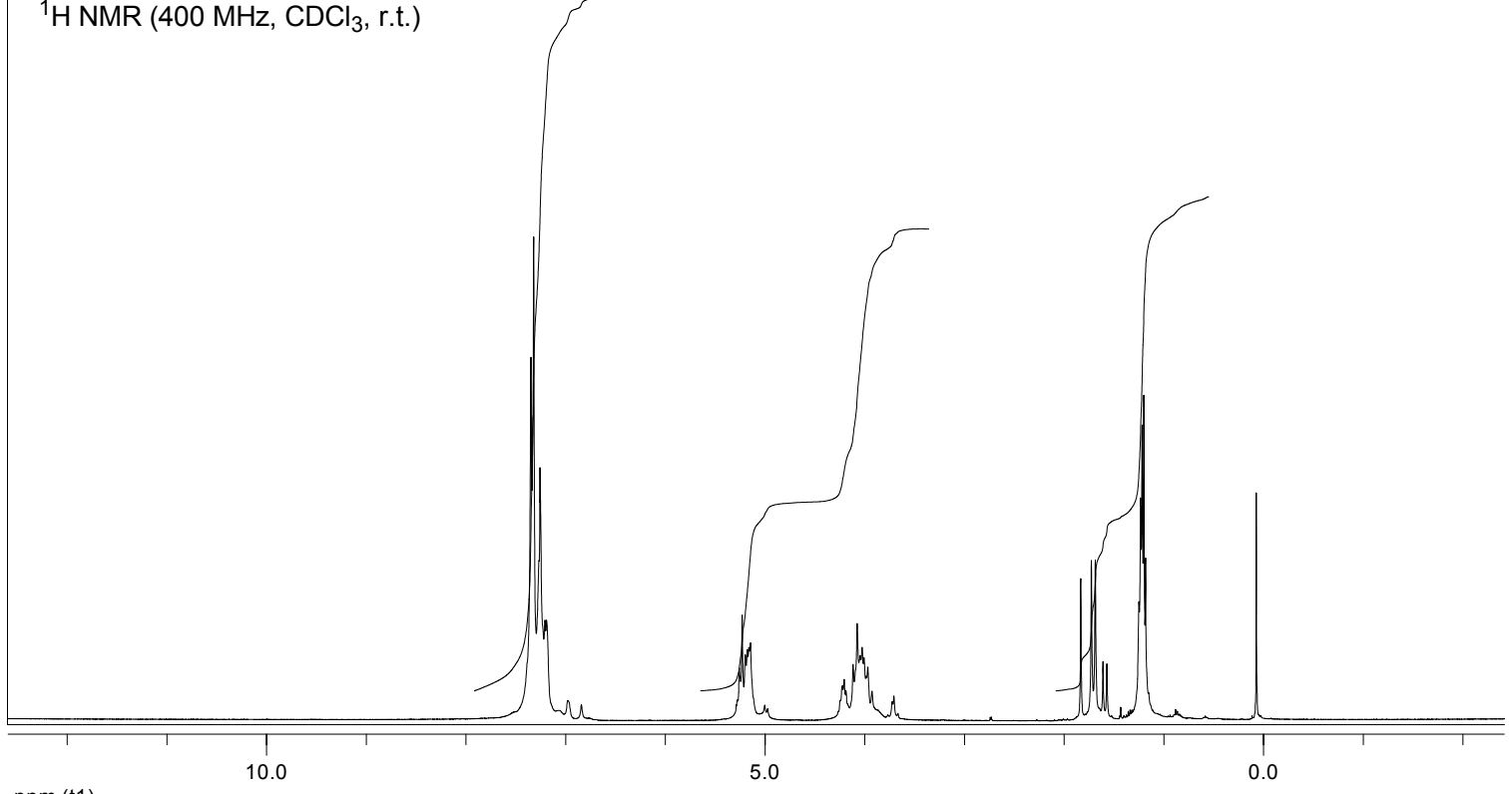

ppm (t1)

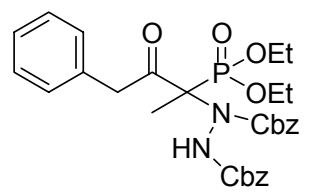

3d

${ }^{1} \mathrm{H}$ NMR $\left(400 \mathrm{MHz}, \mathrm{CDCl}_{3}, 60^{\circ} \mathrm{C}\right)$

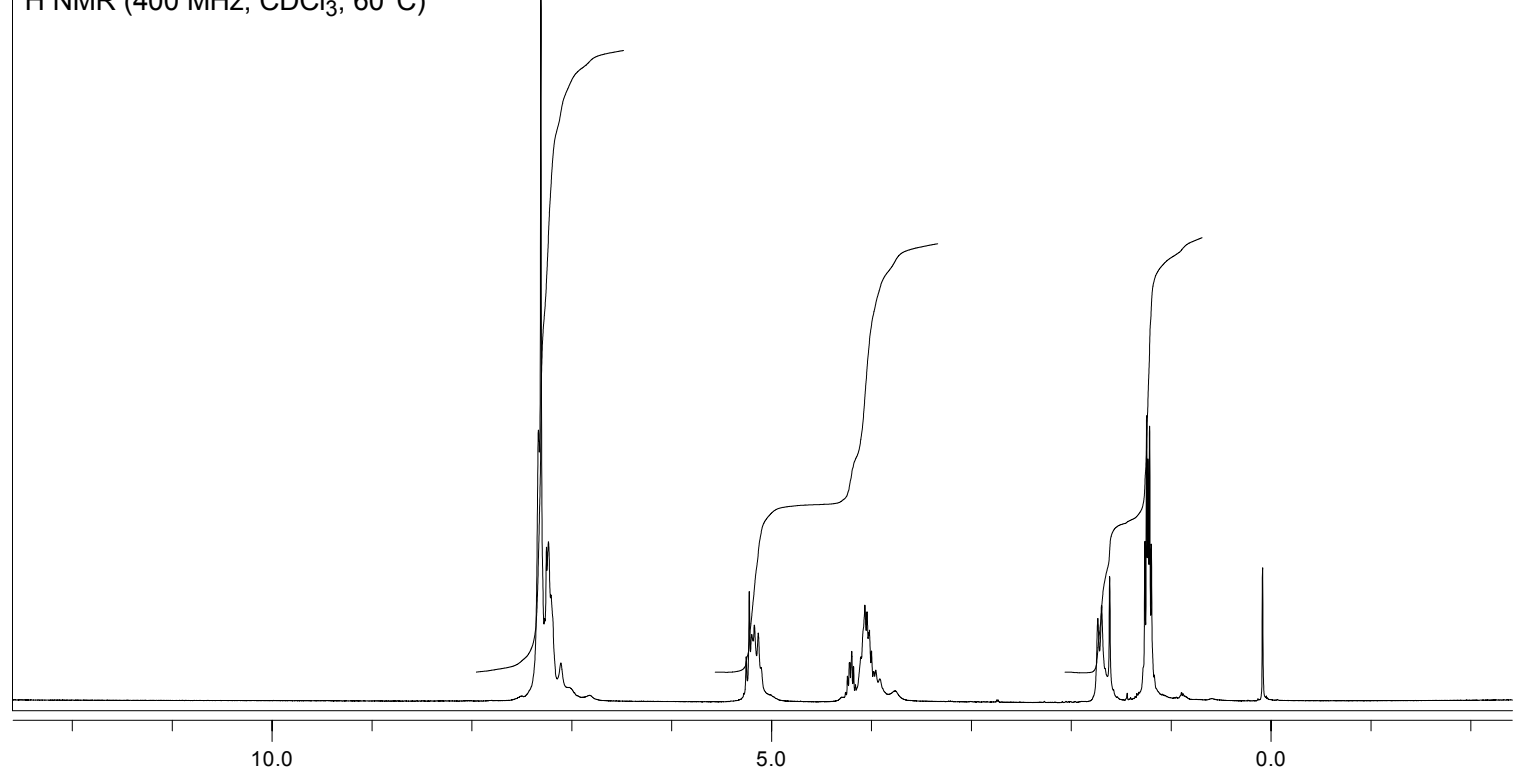

ppm (t1) 


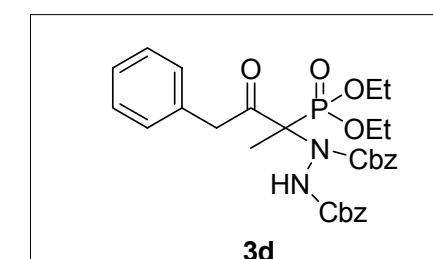

${ }^{13} \mathrm{C} \mathrm{NMR} \mathrm{(100} \mathrm{MHz,} \mathrm{CDCl}_{3}$, r.t. $)$
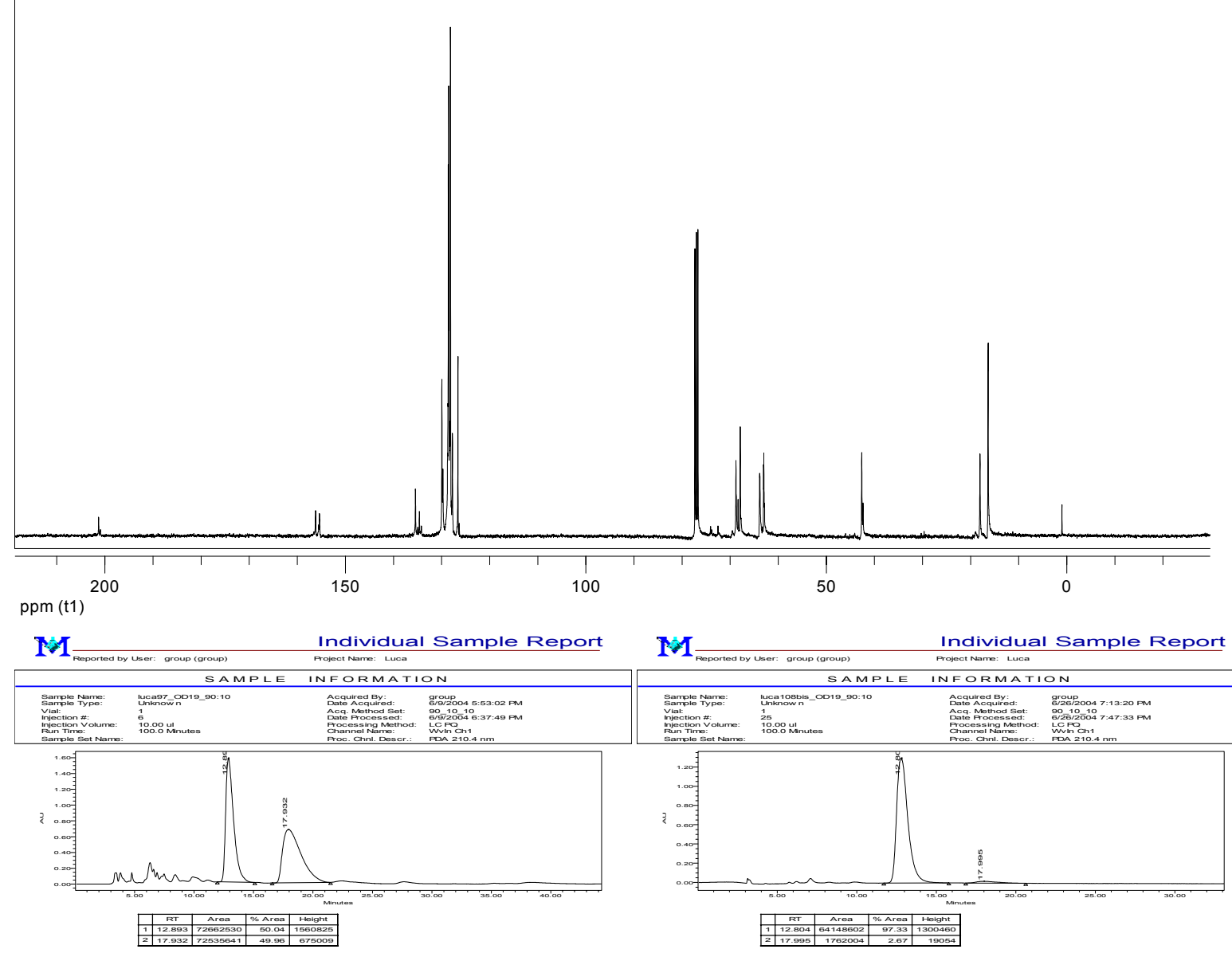

rac-3d
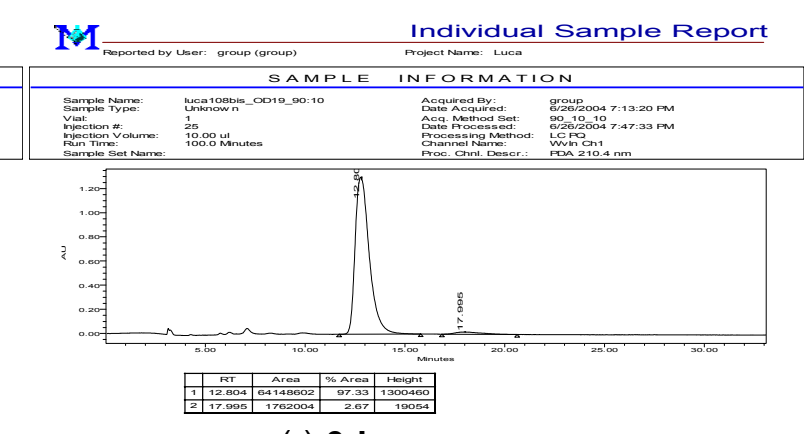

$(-)-3 d$
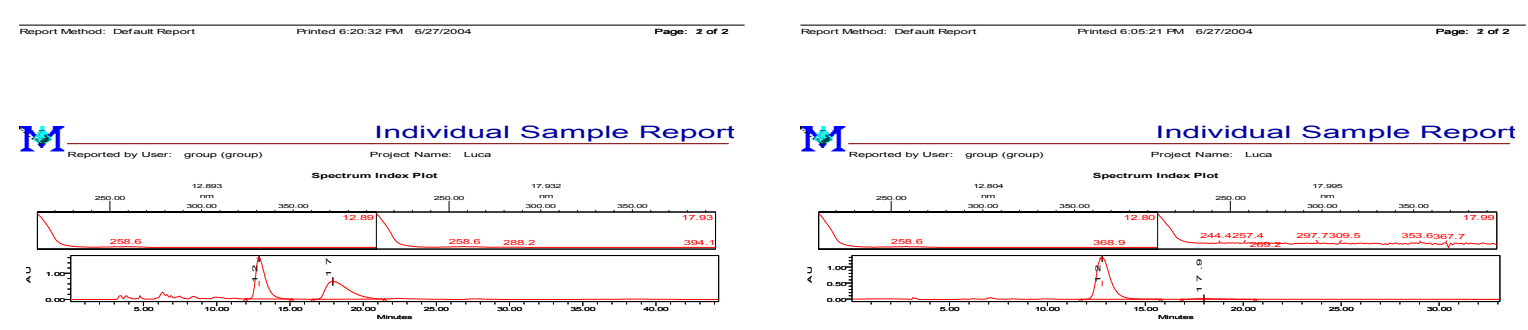

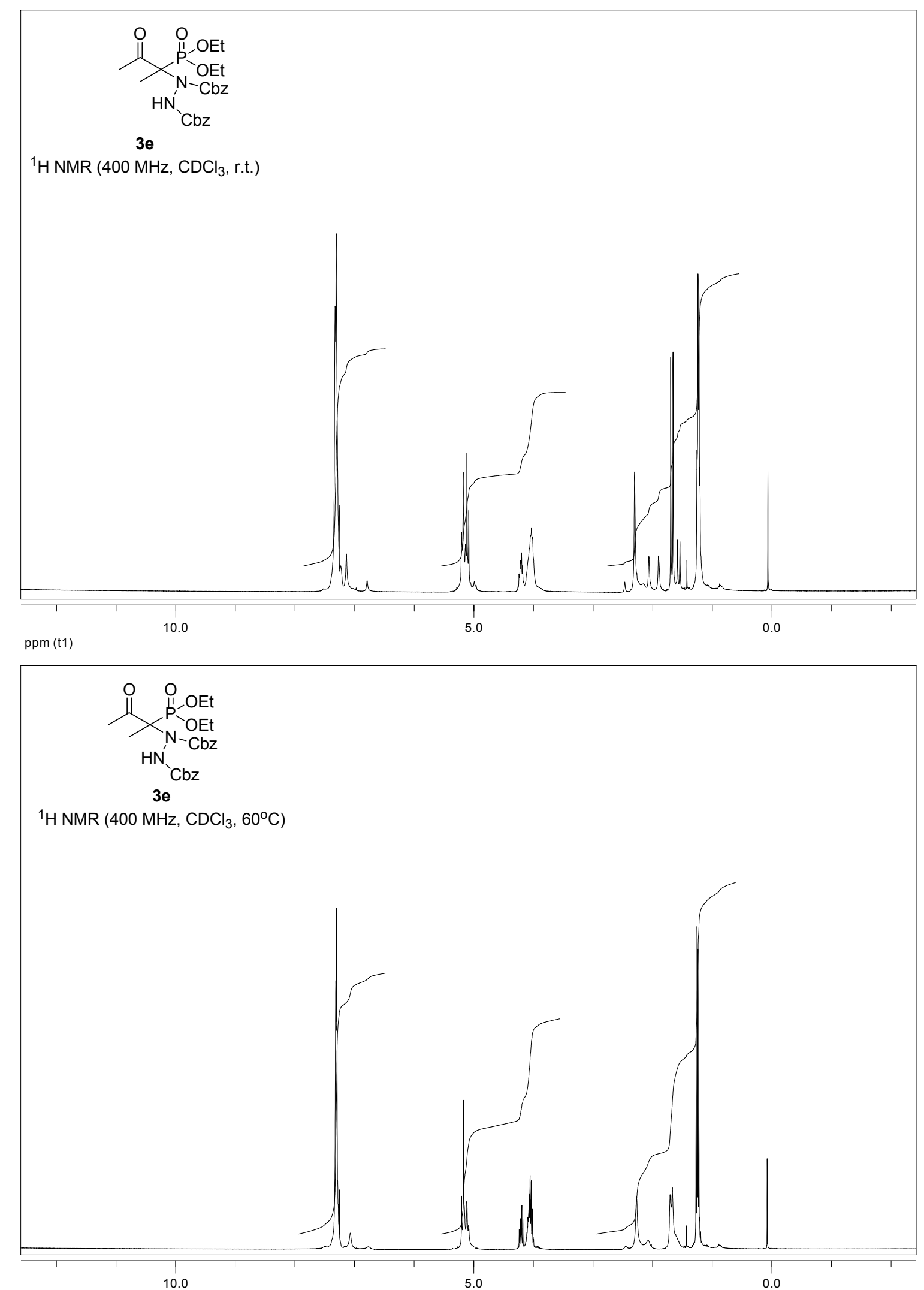

ppm (t1) 


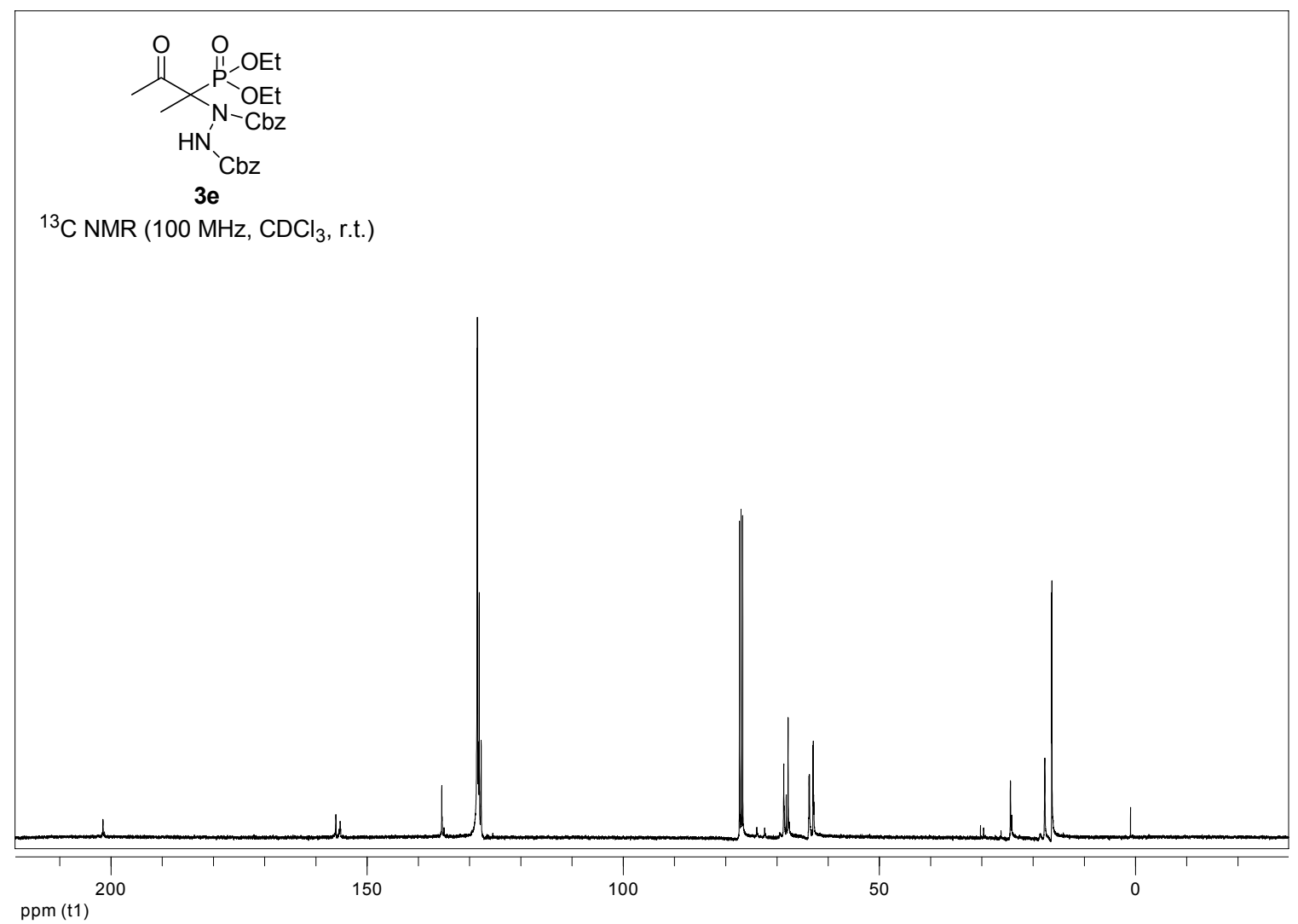




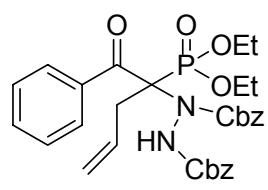

$3 f$

${ }^{1} \mathrm{H}$ NMR (400 MHz, $\mathrm{CDCl}_{3}$, r.t.)
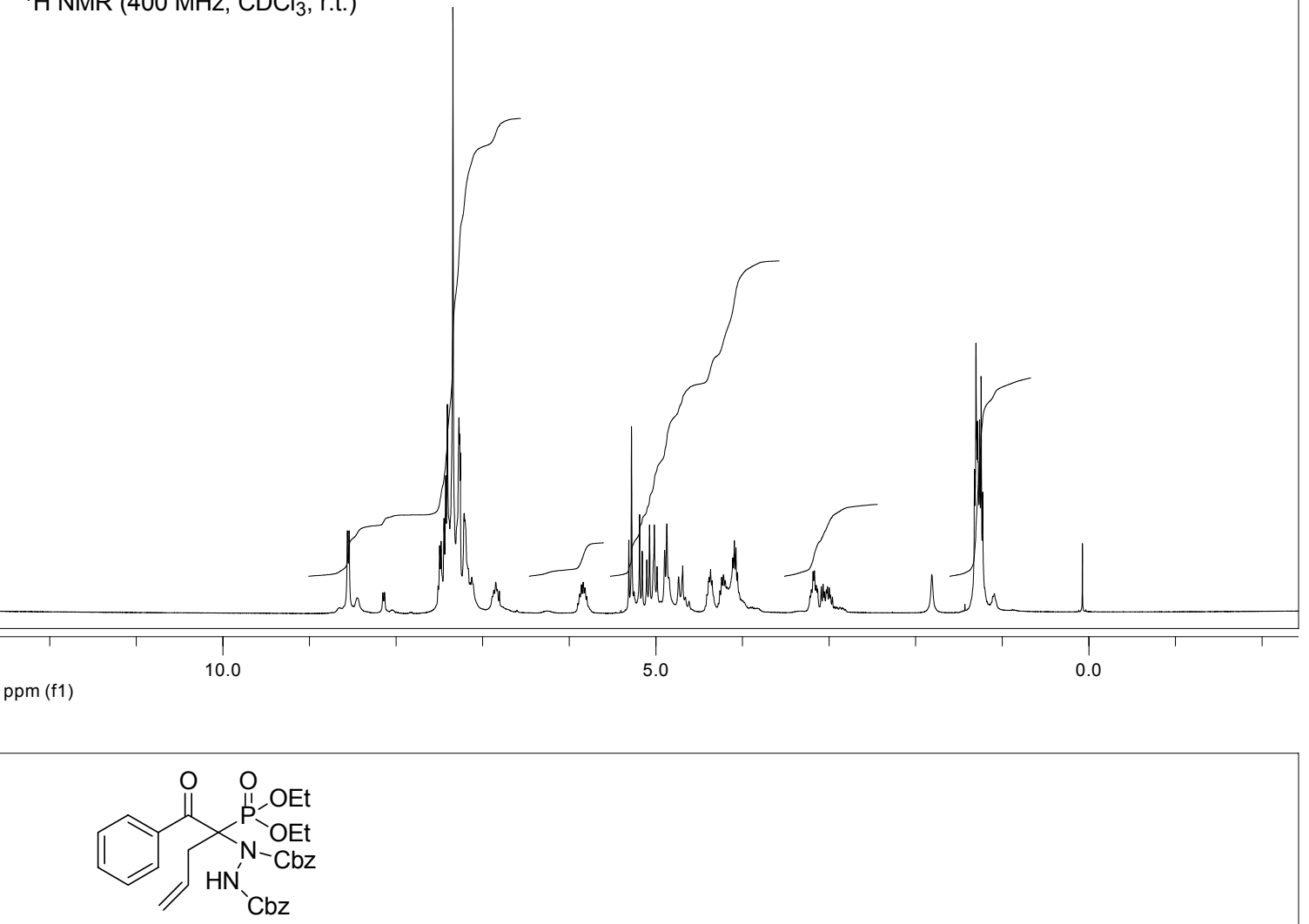

$3 f$

${ }^{1} \mathrm{H}$ NMR $\left(400 \mathrm{MHz}, \mathrm{CDCl}_{3}, 60^{\circ} \mathrm{C}\right)$

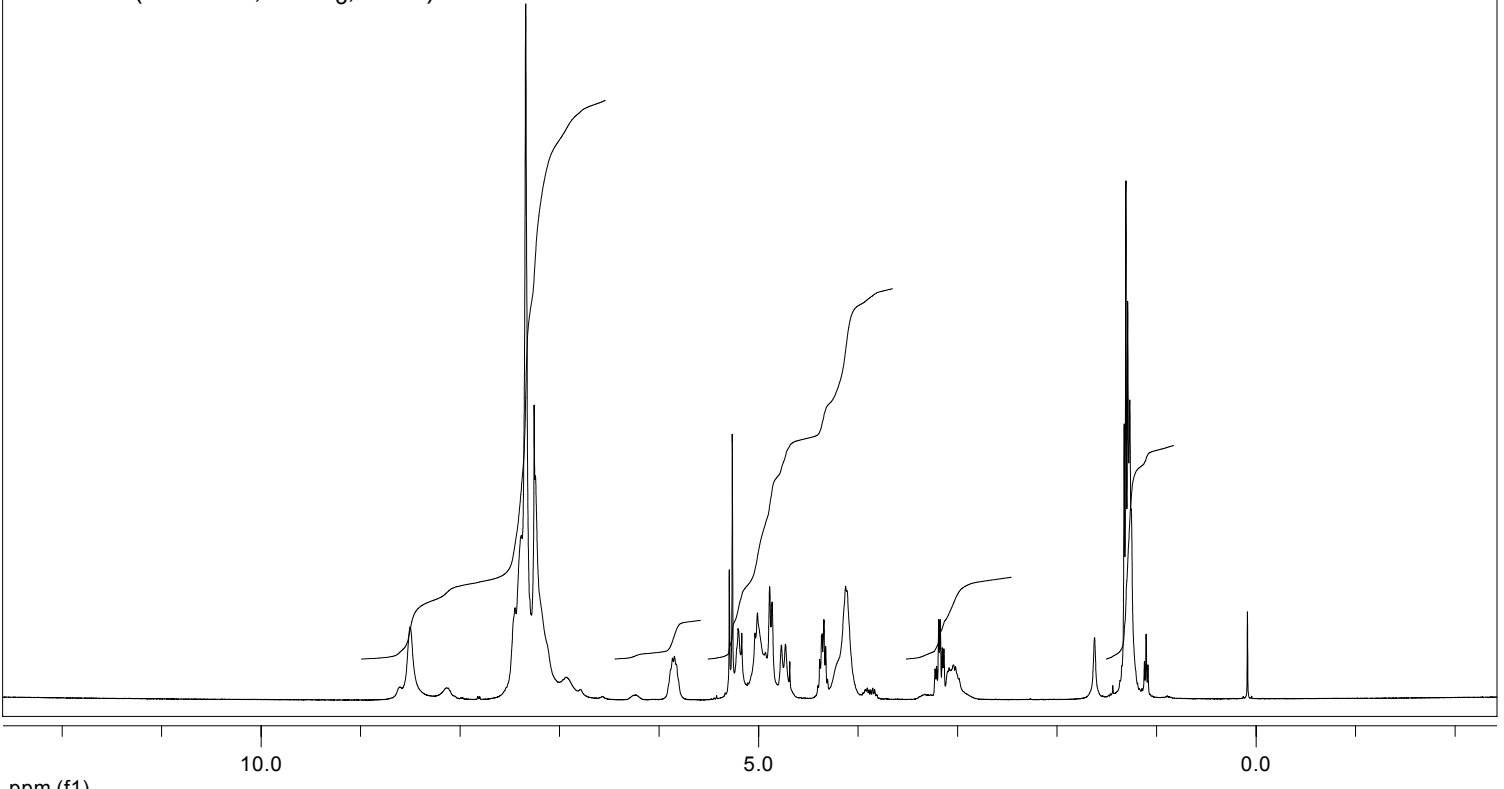

ppm (f1) 


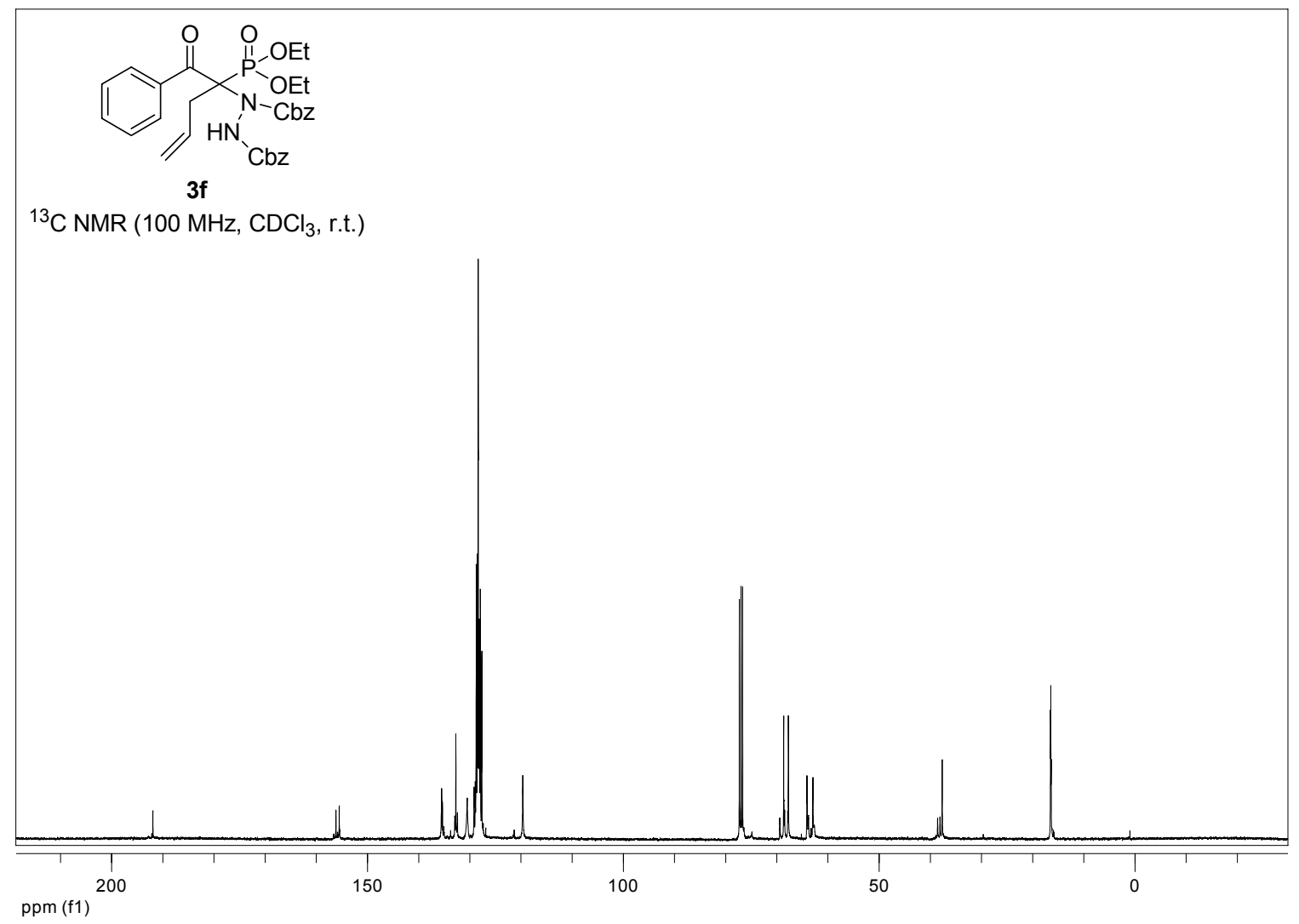




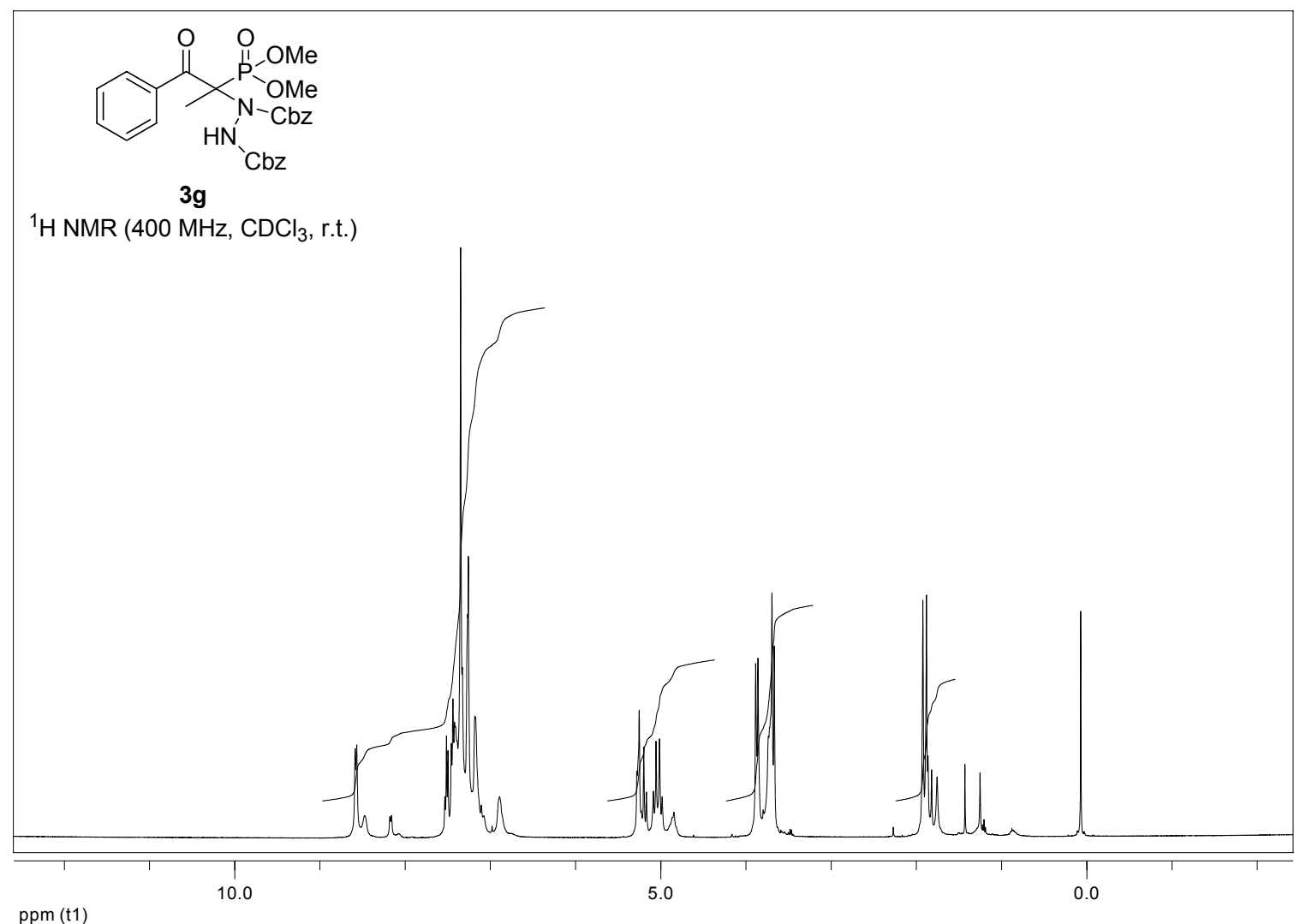

ppm (t1)

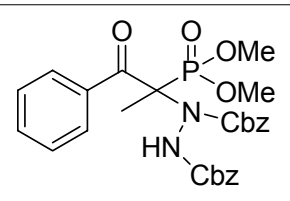

$3 \mathrm{~g}$

${ }^{1} \mathrm{H}$ NMR $\left(400 \mathrm{MHz}, \mathrm{CDCl}_{3}, 60^{\circ} \mathrm{C}\right)$

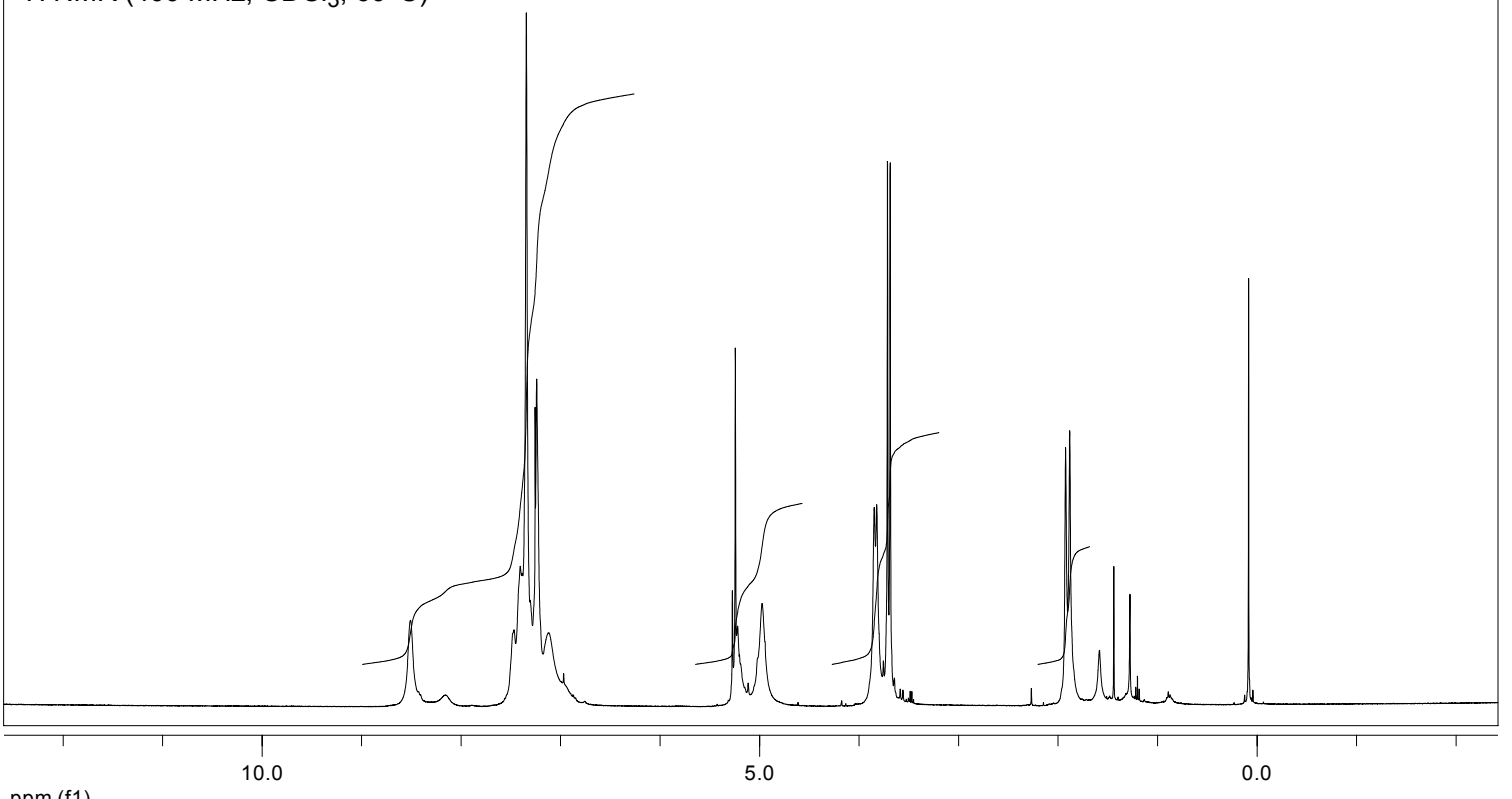

ppm (f1) 


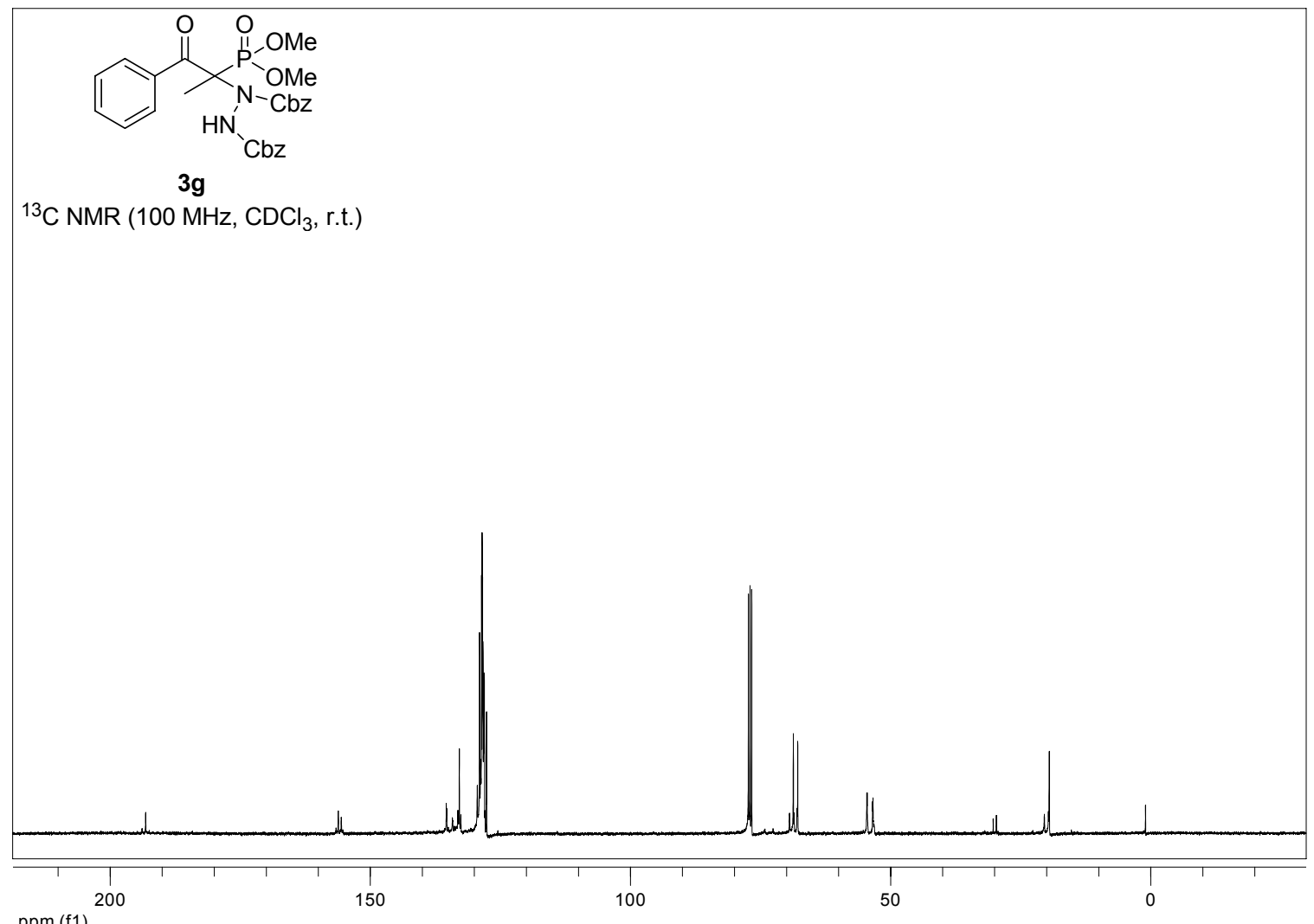

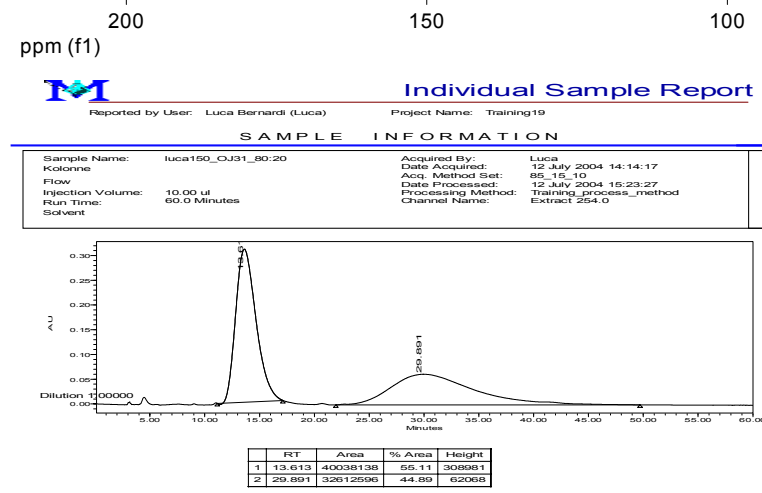

rac-3g
P.1

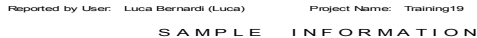

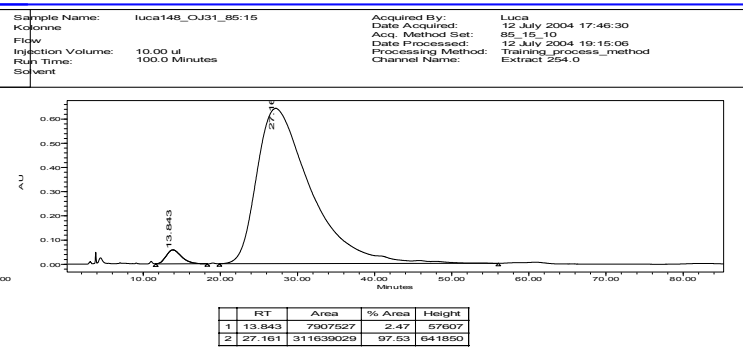

$(-)-3 \mathbf{g}$

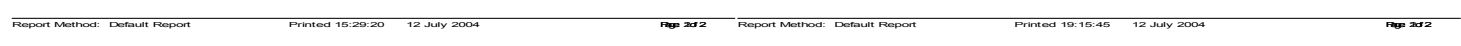

IM
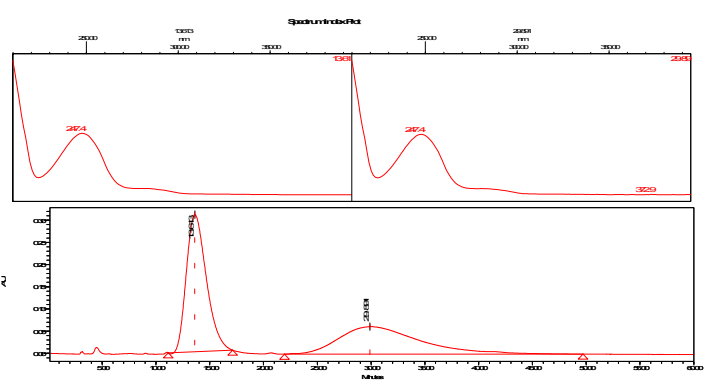

TMI Irohidasenplefapot

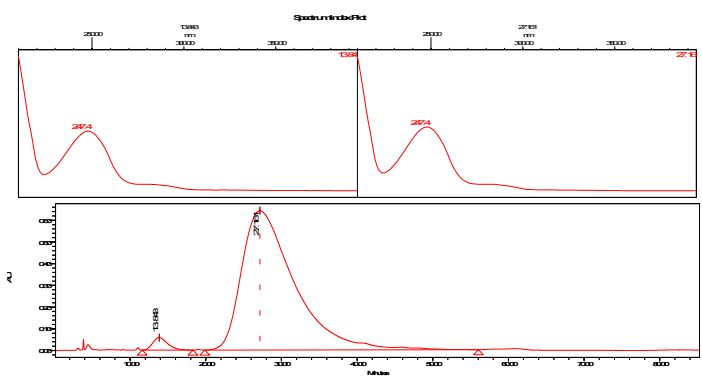

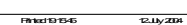




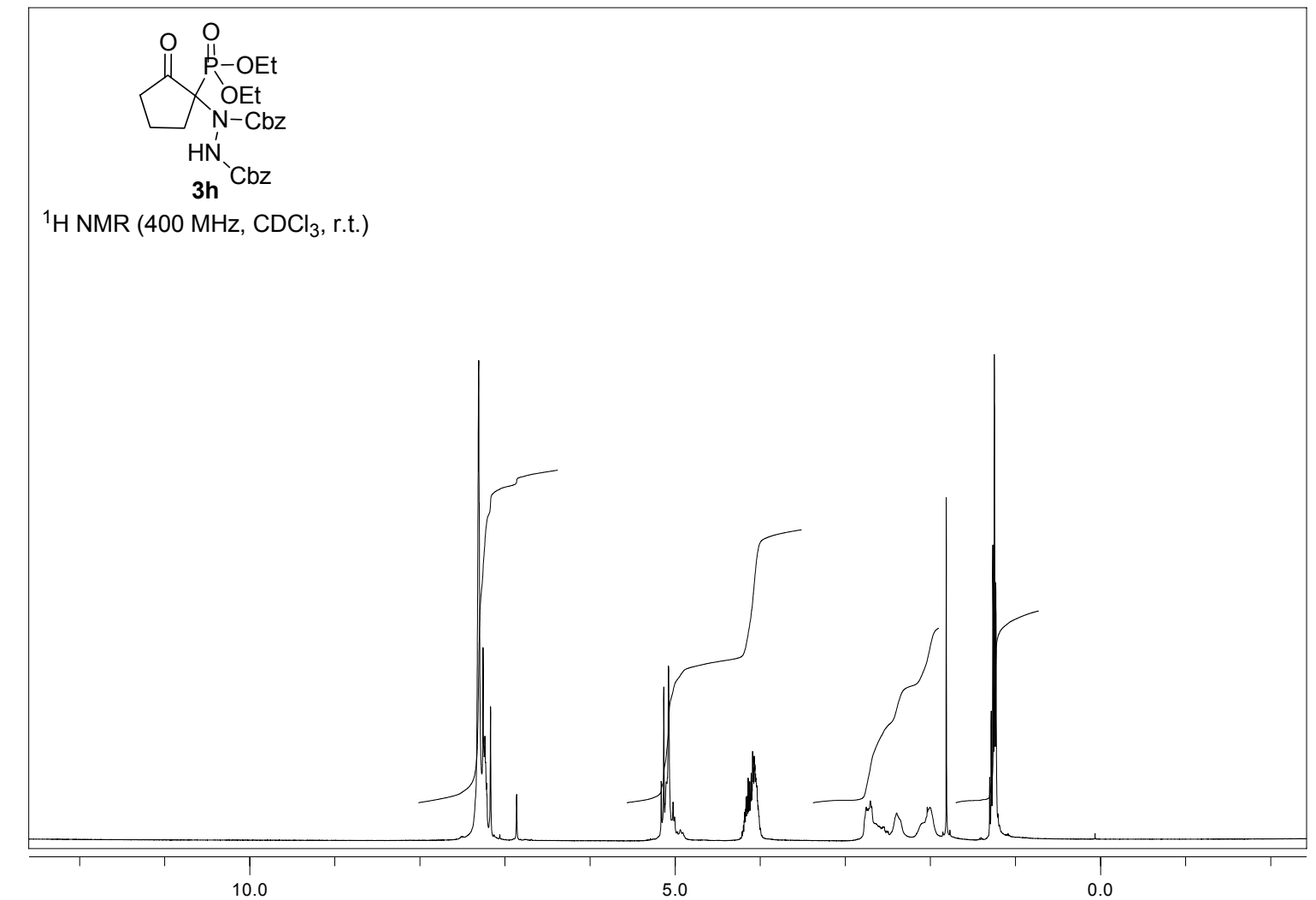

ppm (t1)

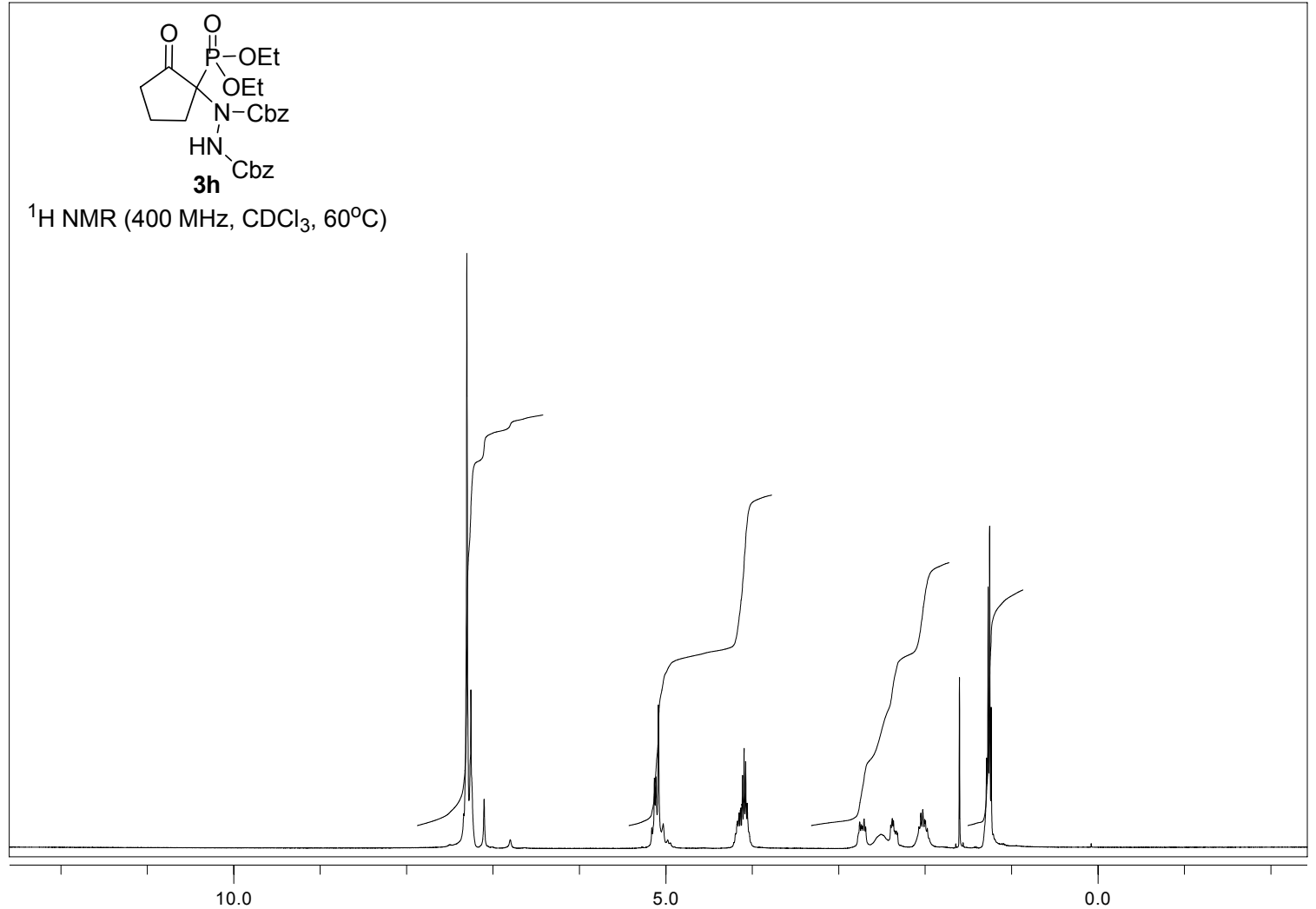

ppm (t1) 


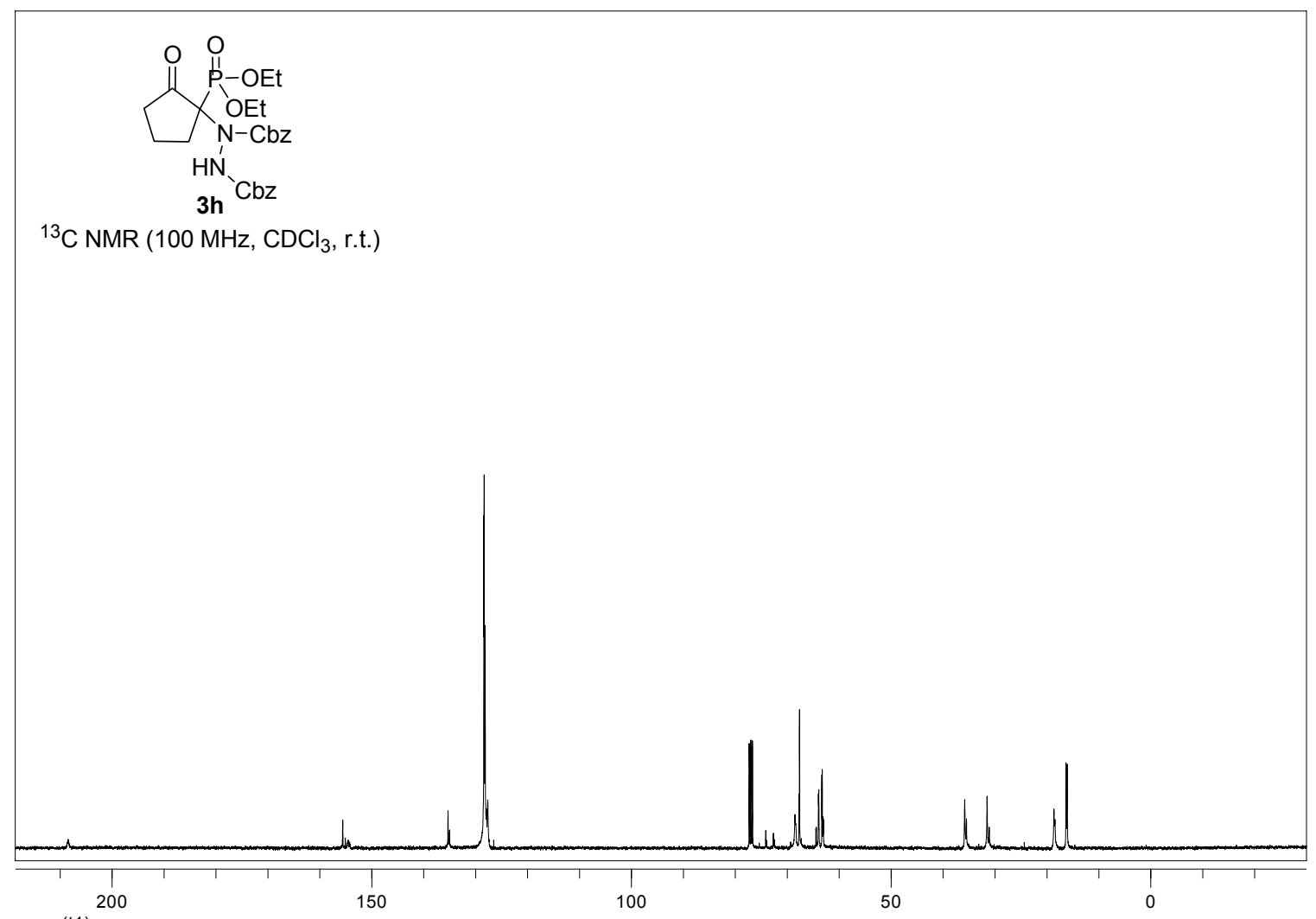

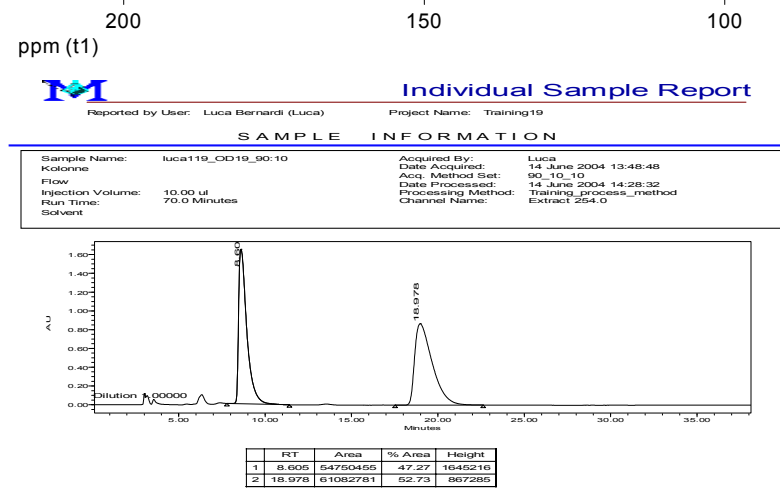

rac-3h

TOI

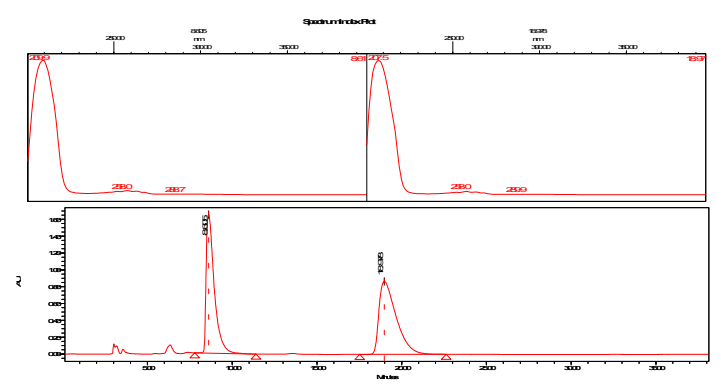

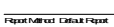
Irahidas Sandefepont

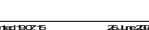

$\mathbf{I}+1$

Individual Sample Report

Project Name: Training19
SAMPLE
S ANFORMATI ON
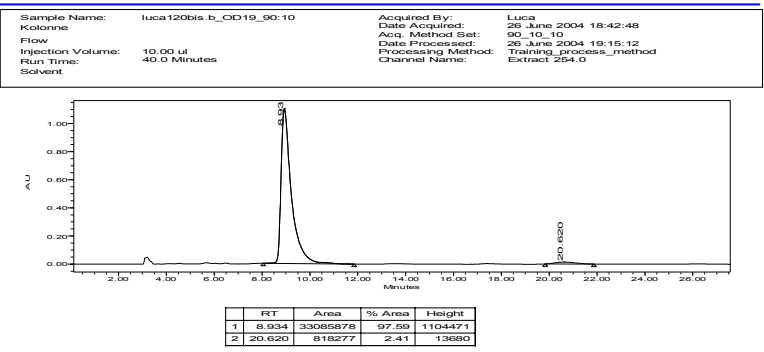

(+)-3h

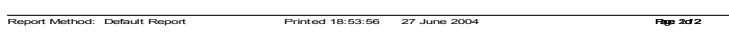

TM Irohidasanderapont

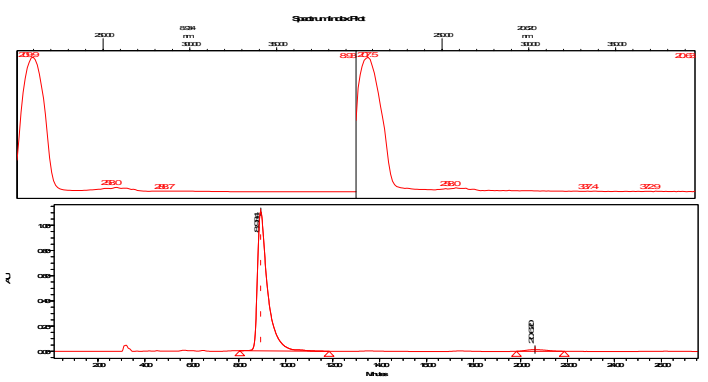

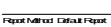



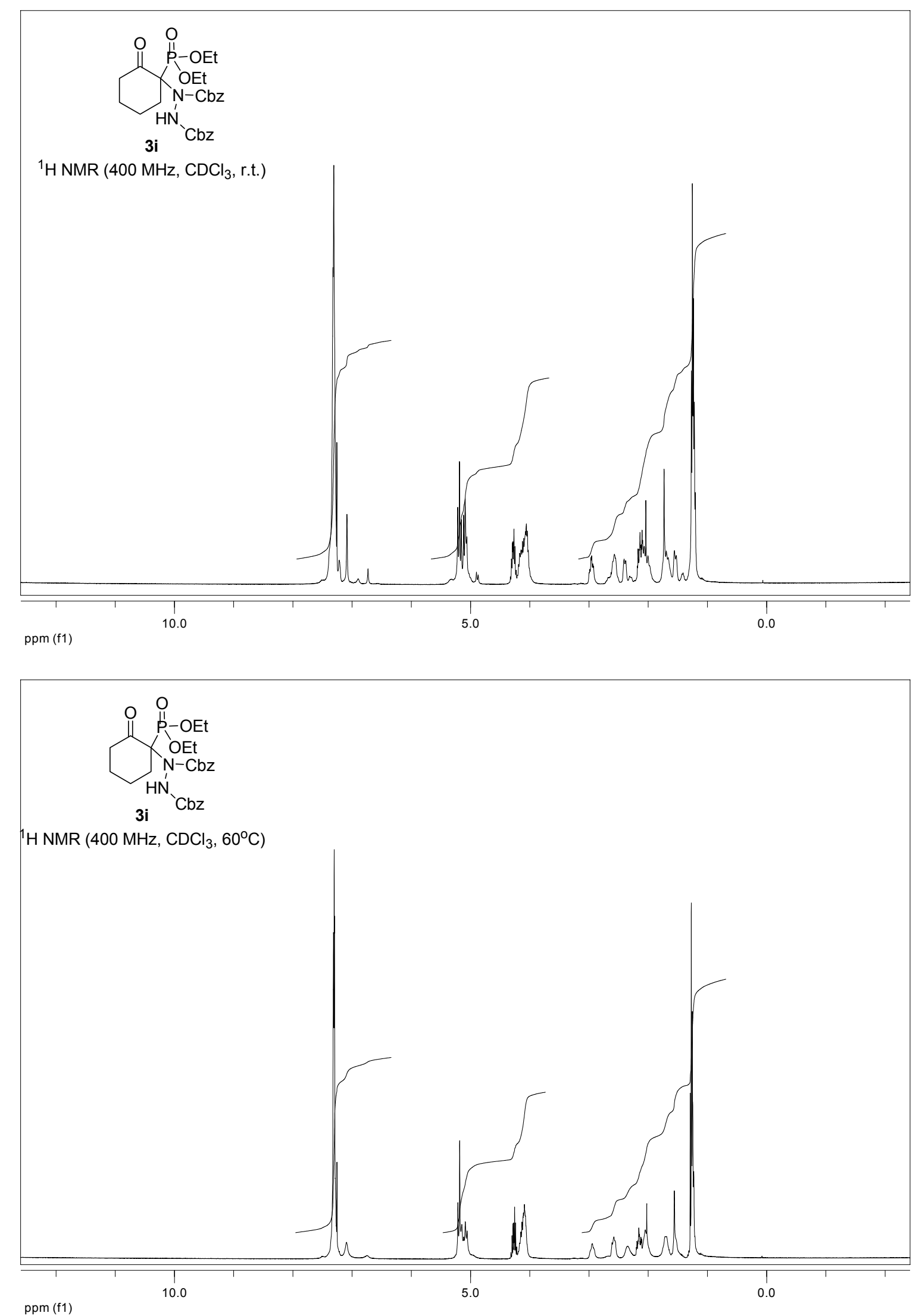

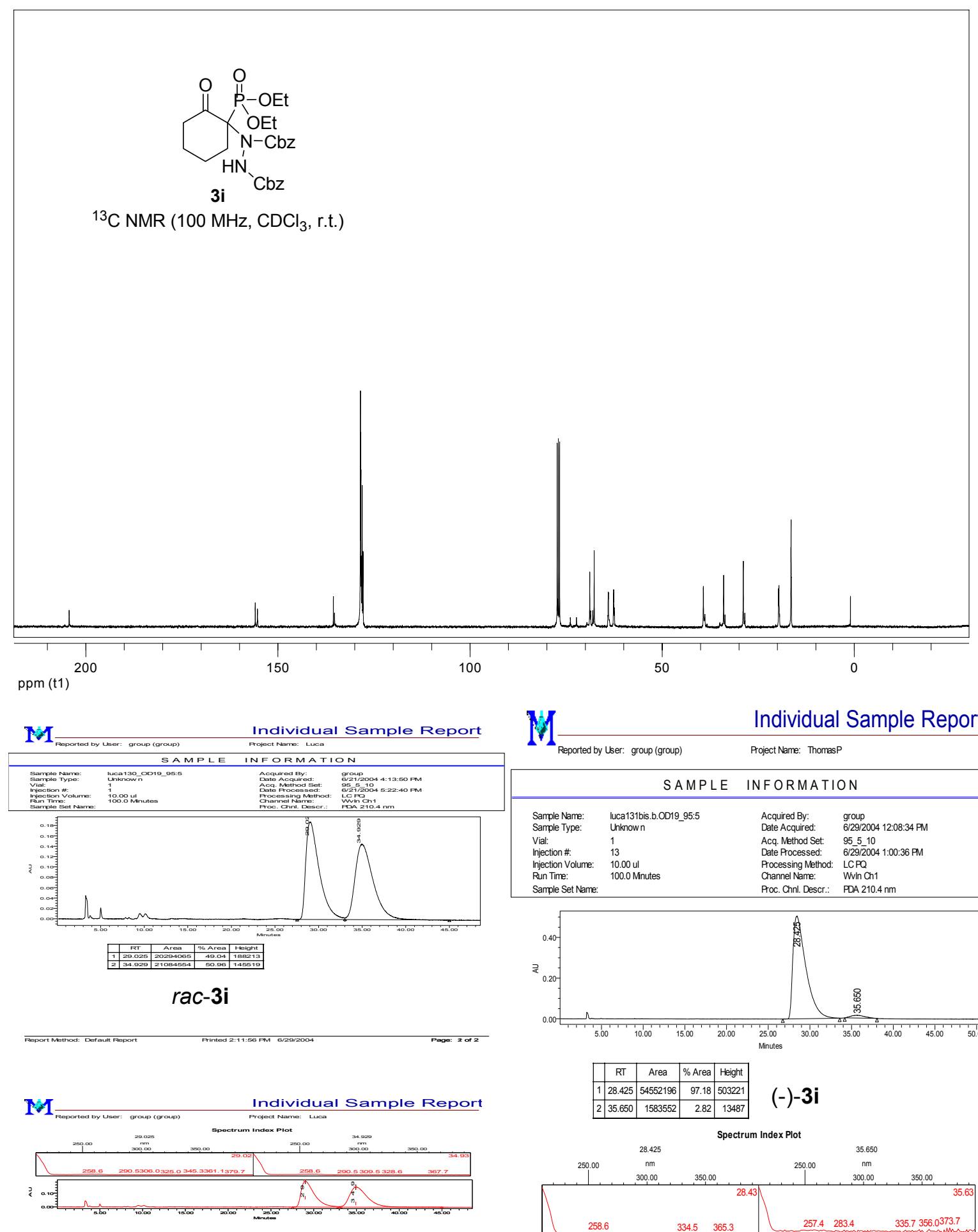

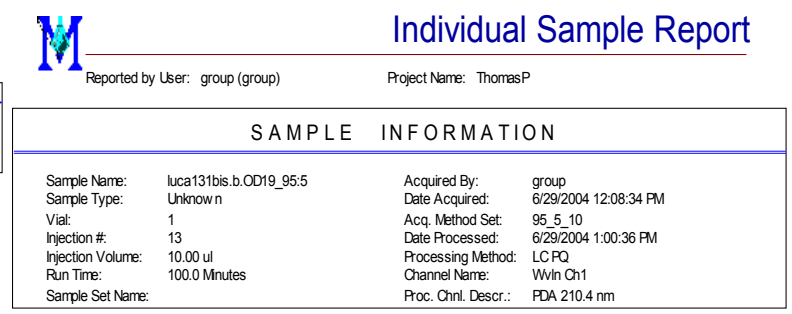
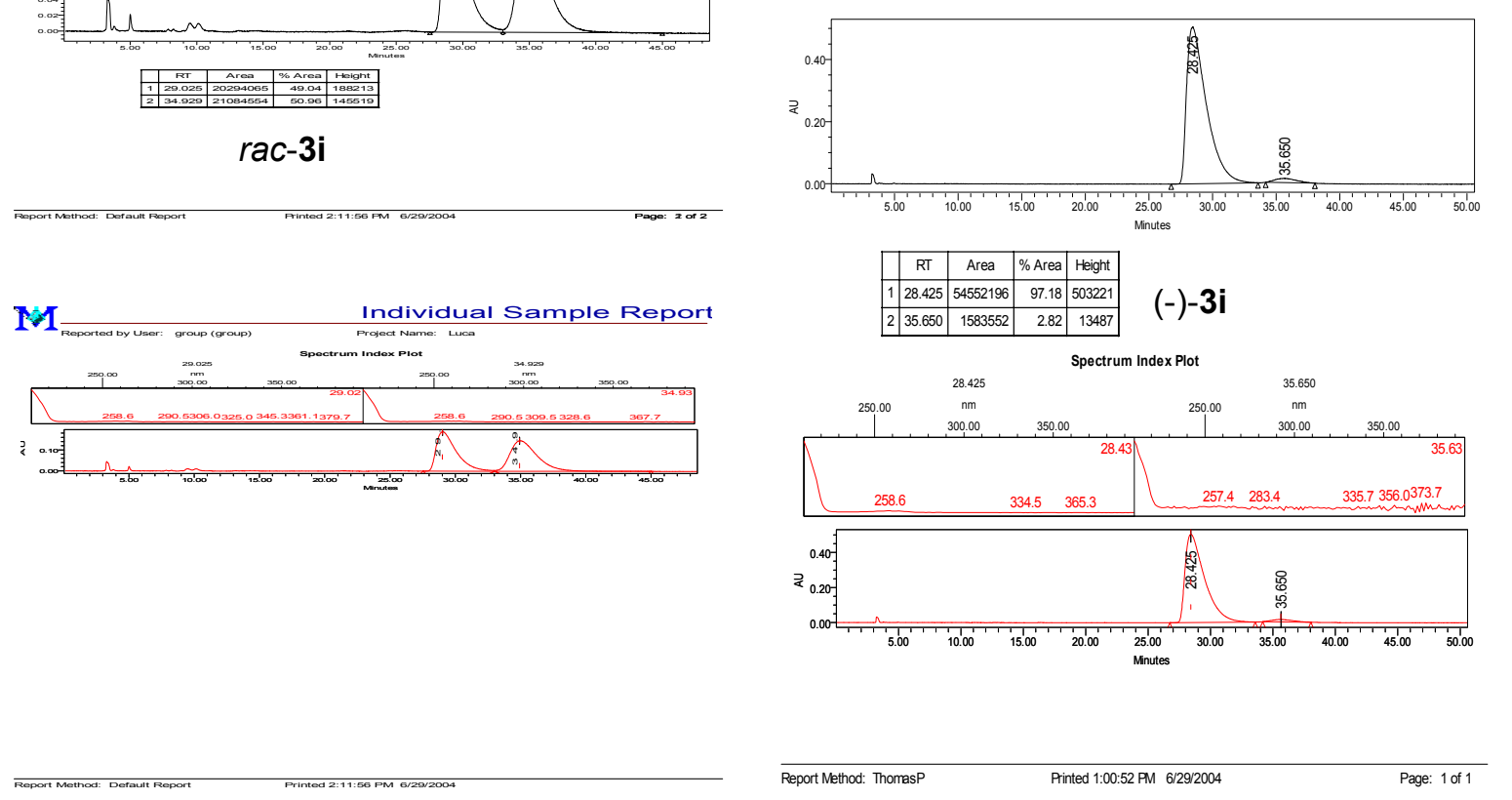

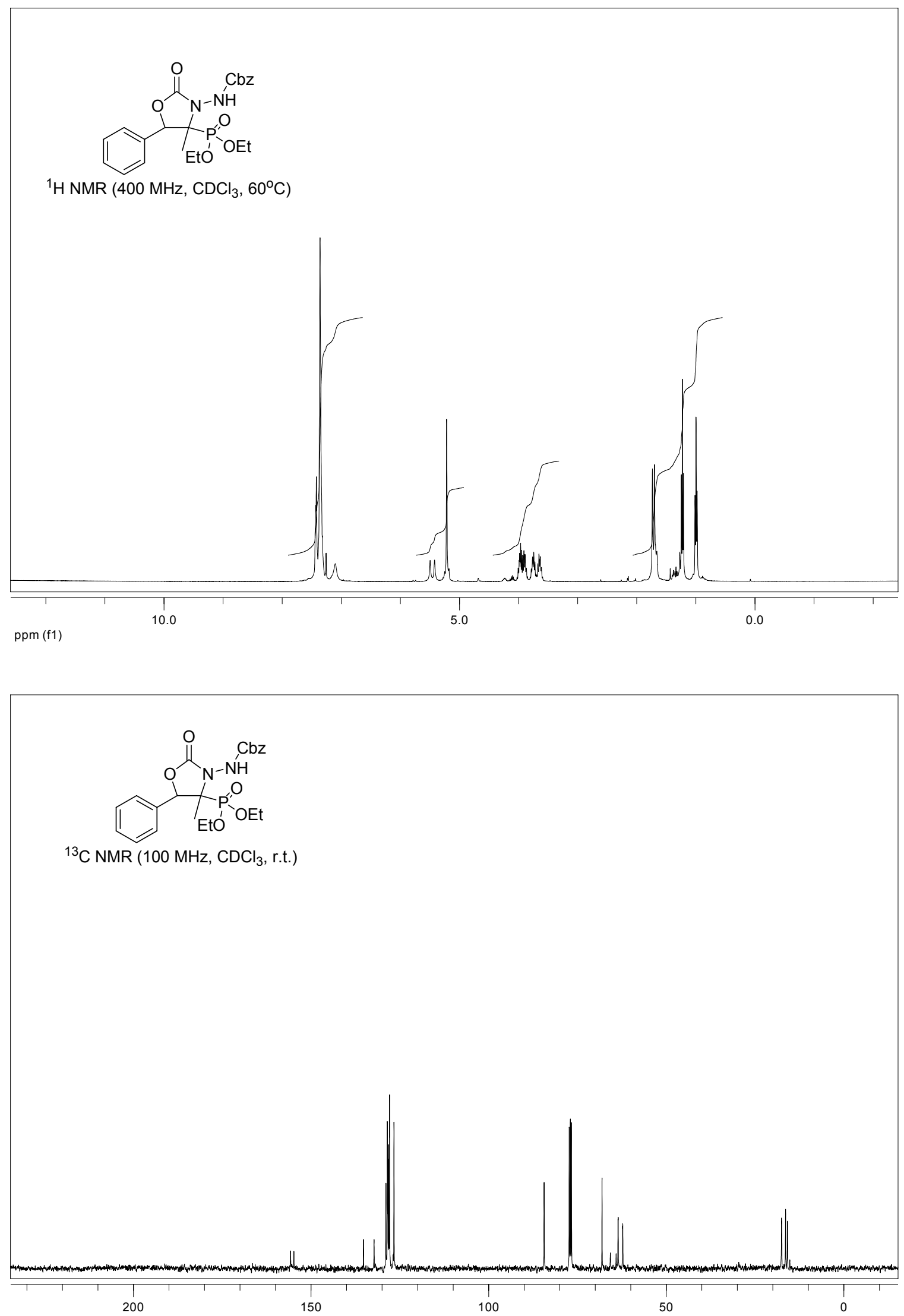

ppm (f1) 


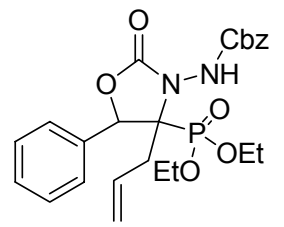

${ }^{1} \mathrm{H}$ NMR $\left(400 \mathrm{MHz}, \mathrm{CDCl}_{3}, 60^{\circ} \mathrm{C}\right)$
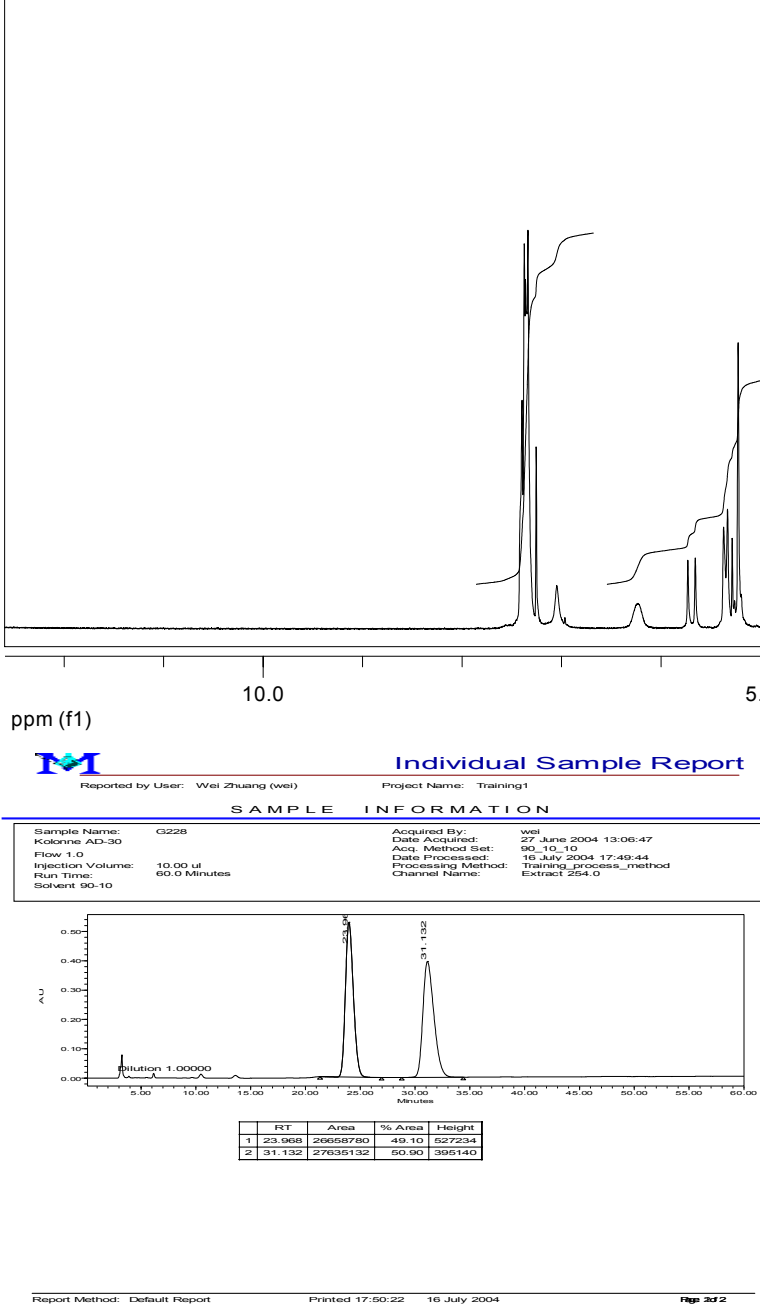

ToI Iravidas Sanderepont

TOI

POI

Wulden

5.0

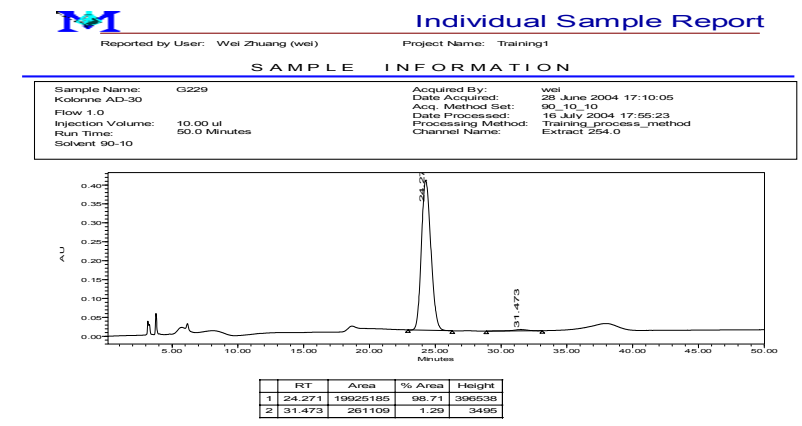

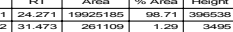

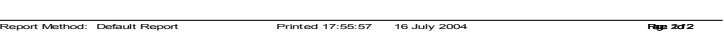

Irenida Sonderazont
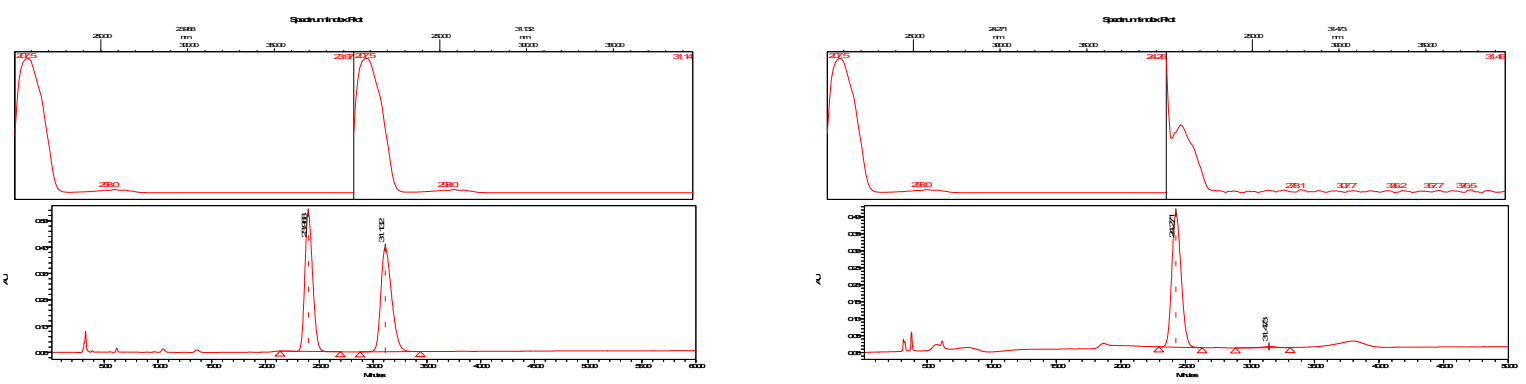

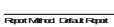

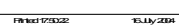

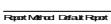

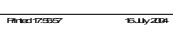



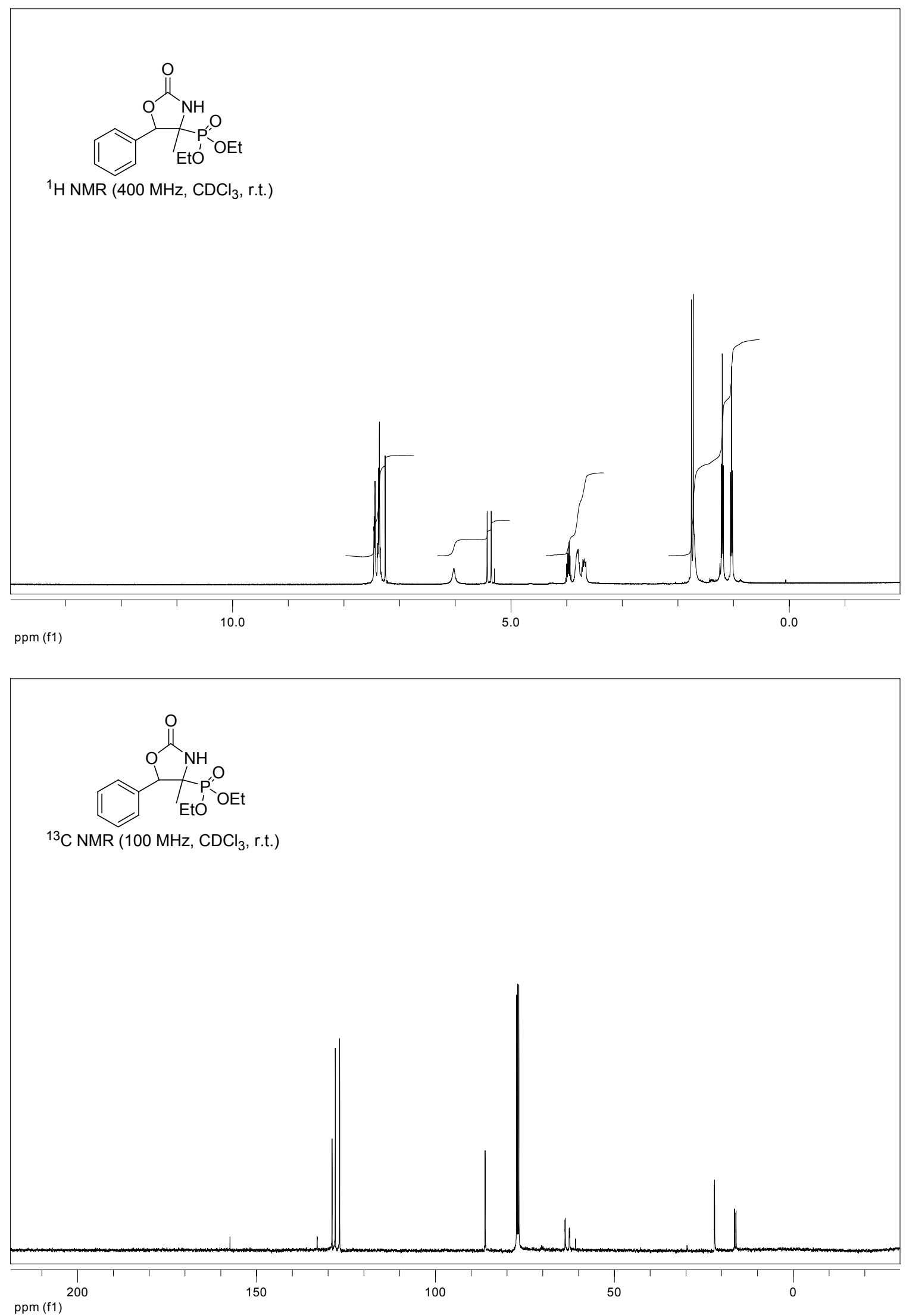

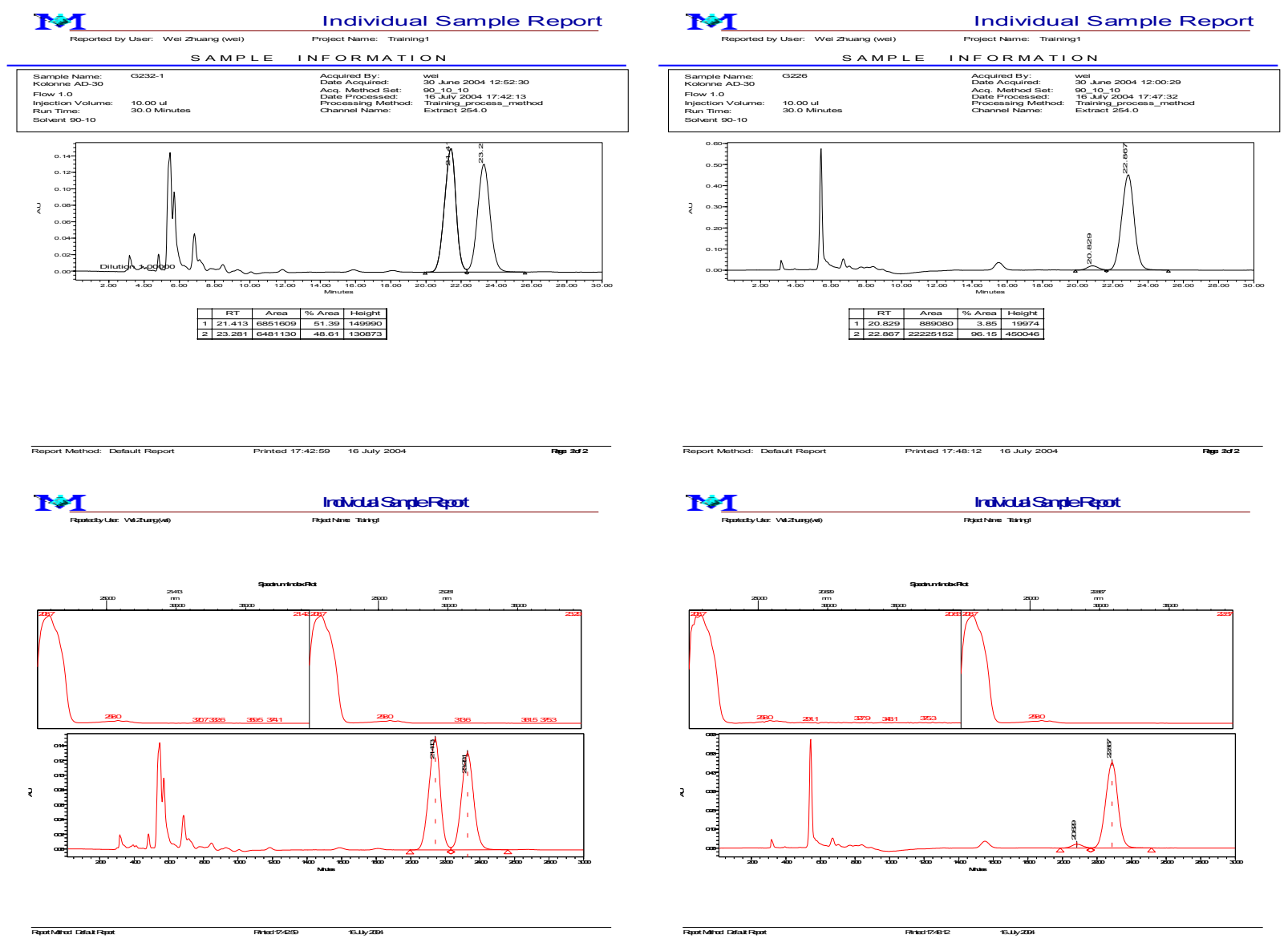
Determination of the absolute configuration of 5. The absolute configuration of $\mathbf{5}$ was determined by the X-ray crystallography of the optically pure [3-(4-chlorobenzoylamino)-4-methyl-2-oxo-5-phenyl-oxazolidin-4-yl]-phosphonic acid diethyl ester 6.

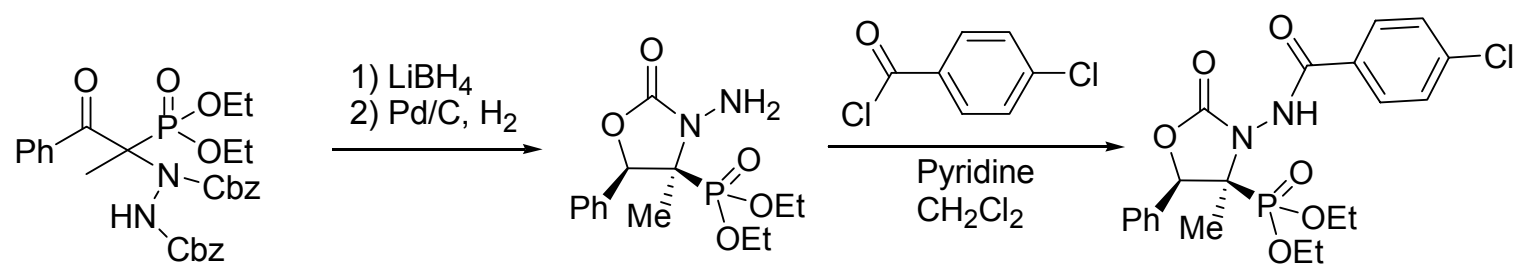

$3 \mathbf{b}$

6

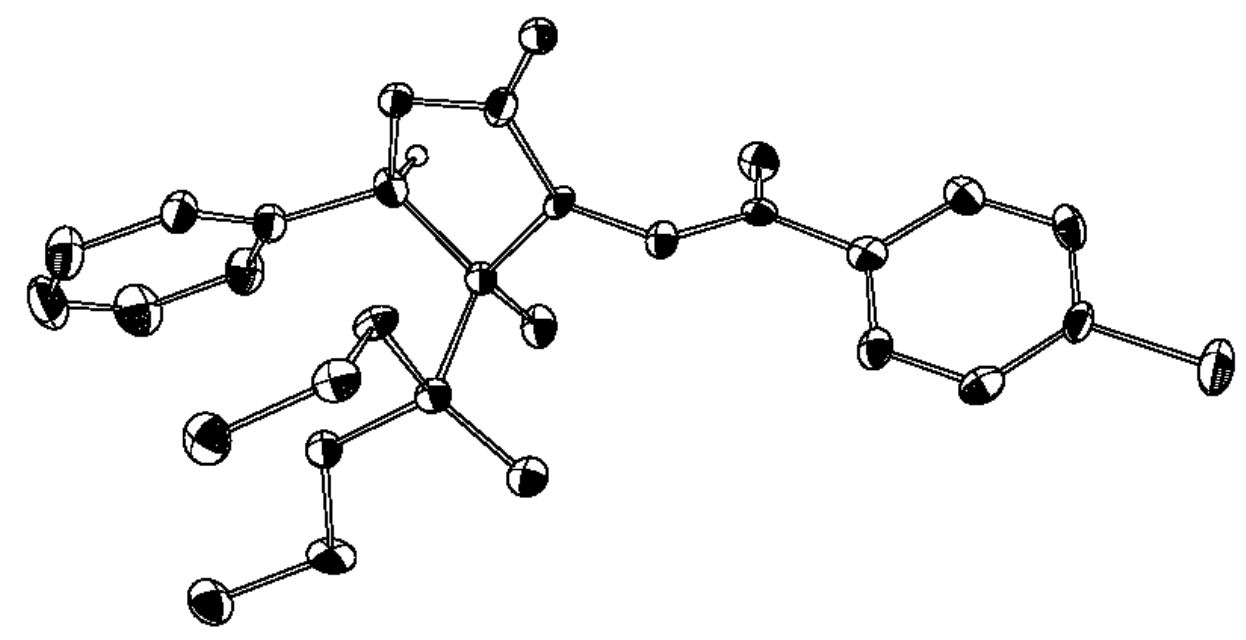

X-Ray work: Crystals of, $\mathrm{C}_{21} \mathrm{H}_{24} \mathrm{ClN}_{2} \mathrm{O}_{6} \mathrm{P}$, are monoclinic, $\mathrm{P} 2{ }_{1}$, with unit cell:a $=$ 10.5320(9) $\AA, b=7.3532(7) \AA, c=15.228(1) \AA, V=1107.67(16) \AA^{3}, Z=2$. Data were collected at $100 \mathrm{~K}$ on a SMART diffractometer with CCD detector. The structure was solved by direct methods and refined by least squares methods to final $\mathrm{R}=0.044, \mathrm{Rw}=$ 0.049 , GOF $=0.942$ using 3199 reflections with $\mathrm{I}>3 \sigma(\mathrm{I}), 280$ parameters refined. Least squares refinement included a parameter according to Rogers ${ }^{4}$ refined against all 3199 significant reflections including 749 Bijvoet pairs, determining the chirality.

\footnotetext{
${ }^{4}$ Rogers, D. Acta Cryst. 1981, A37, 734.
} 
Proposed intermediate for the enantioselective approach of the amination reagent.

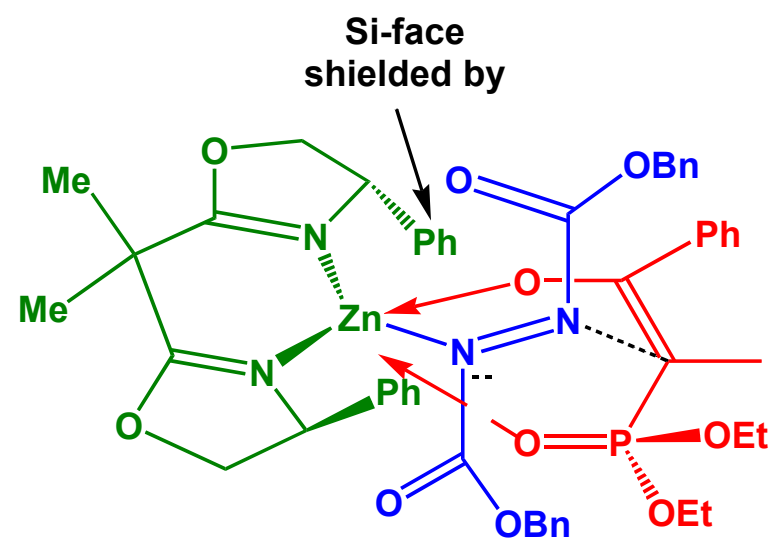

